\title{
Event-Triggered State Estimation for Discrete-Time Multi-Delayed Neural Networks with Stochastic Parameters and Incomplete Measurements
}

\author{
Bo Shen, Zidong Wang and Hong Qiao
}

\begin{abstract}
In this paper, the event-triggered state estimation problem is investigated for a class of discrete-time multi-delayed neural networks with stochastic parameters and incomplete measurements. In order to cater for more realistic transmission process of the neural signals, we make the first attempt to introduce a set of stochastic variables to characterize the random fluctuations of system parameters. In the addressed neural network model, the delays among the interconnections are allowed to be different, which are more general than those in existing literature. The incomplete information under consideration includes randomly occurring sensor saturations and quantizations. For the purpose of energy saving, an event-triggered state estimator is constructed and a sufficient condition is given under which the estimation error dynamics is exponentially ultimately bounded in the mean square. It is worth noting that the ultimate boundedness of the error dynamics is explicitly estimated. The characterization of the desired estimator gain is designed in terms of the solution to a certain matrix inequality. Finally, a numerical simulation example is presented to illustrate the effectiveness of the proposed event-triggered state estimation scheme.
\end{abstract}

Index Terms-Event-triggered state estimation; exponentially ultimate boundedness; incomplete measurements; neural networks; quantizations; sensor saturations; stochastic parameters.

\section{INTRODUCTION}

In the past few years, the dynamical analysis problem of various neural networks has stirred a great deal of research interest, and a rich body of research results has been reported in the literature. For example, in [10], [23], [24], [32], [40], [45], [46], the stability and synchronization issues have been investigated for different kinds of neural networks. After decades of constant developments, the context of neural networks has gone far beyond the traditional biological neural networks. Nowadays, artificial neural networks have been

This work was supported in part by the National Natural Science Foundation of China under Grants 61473076, 61329301, 61210009 and 61134009, the Shu Guang project of Shanghai Municipal Education Commission and Shanghai Education Development Foundation under Grant 13SG34, the Program for Professor of Special Appointment (Eastern Scholar) at Shanghai Institutions of Higher Learning, the Fundamental Research Funds for the Central Universities, and the DHU Distinguished Young Professor Program.

B. Shen is with the School of Information Science and Technology, Donghua University, Shanghai 201620, China. (Email: bo.shenedhu.edu.cn)

Z. Wang is with the Department of Computer Science, Brunel University London, Uxbridge, Middlesex, UB8 3PH, United Kingdom. (Email: Zidong. Wang@brunel.ac.uk)

H. Qiao is with the State Key Lab of Management and Control for Complex Systems, Institute of Automation, Chinese Academy of Sciences, Beijing 100190, China and also with the CAS Centre for Excellence in Brain Science and Intelligence Technology (CEBSIT), Shanghai 200031, China. widely applied in a variety of research domains including statistical signal processing [21], [39], pattern recognition [1], [8], intelligent data analysis [16], [19], robotics and control [2], [17], where the conventional meaning of the neurons has been extended from biological ones to those nodes having adaptive weights for approximating nonlinear functions of their inputs. For example, the "neurons" in a recurrent neural network could be a computing unit as long as it is capable of biophysical simulation and neuromorphic computing, and a network of such computing units holds therefore the advantages of approximation, learning as well as adaption [12]. Depending on the scale of the networked artificial neurons, the full states of certain primary neurons are vitally important for achieving certain tasks (e.g. real-time tracking in robotics). Unfortunately, it is often the case that the states of such neurons are not immediately available and we are only able to make a series of observations (e.g. measurement outputs) transmitted through channels (e.g. in a remote network of limited bandwidth) subject to communication constraints. In this case, the network-induced phenomena (e.g., packet dropout, saturation and quantization) would pose great challenges to the state estimation issues of the artificial neural networks.

In order to describe the intermittent measurements, considerable research effort has been made and a variety of measurement models have been proposed to reflect the networkinduced phenomena, see, e.g. [5], [11], [15], [20], [26], [29], [35], [41], [43], where most measurement models are capable of representing one or two phenomena of the intermittent measurements. However, in the real communication environments, more network-induced phenomena could take place simultaneously and, therefore, it is of great necessity to look for a novel measurement model that can reflect as more phenomena as possible in a unified way. To this end, in [33], a unified measurement model has been established by using a Kronecker delta function and, based on it, the $H_{\infty}$ state estimation problem has been investigated for complex networks subject to randomly occurring sensor saturations, quantizations and missing measurements. For neural networks, recently, some results have appeared on the state estimation problem with intermittent measurements. Nevertheless, the state estimation problem for more general neural networks with more realistic network-induced phenomena has not yet received adequate research attention.

Time delays serve as an inherent characteristic in the implementation process of neural networks which may cause the system to oscillate. In the past few decades, a great number 
of papers have been published on the neural networks with various time-delays such as constant or time-varying delays [36], distributed delays [38] and mixed time-delays [27]. With respect to the state estimation problem, there have also been a lot of results available in the existing literature. For example, in [3], [18], [36], [44], the state estimation problems have been discussed for the continuous-time delayed neural networks and, in [31], the similar results have been obtained for the discrete-time case. Note that the time delays considered above are simply assumed to be identical when the information is transmitted from one neuron to others. Actually, in the communication among the neurons, the occurred delays may be different since the distances from the certain neuron to others are different. Of course, the existence of such multidelays may complicate the analysis and design of systems, especially for the performance analysis of the estimation error in the state estimation problems. This is why, to date, very little attention has been paid to the state estimation problem for neural networks with multi-delays and this is the first motivation in our paper to handle such a problem.

Recently, event-triggered control and estimation schemes have been a popular research topic in the control community. Different from the traditional time-triggered scheme, in the event-triggered strategy, the controller or estimator is modulated only when a certain triggering condition is met, which can effectively reduce the unnecessary energy consumption. Energy saving is particularly important in those resourcelimited environments. For example, in multi-agent systems, due to low communication bandwidth and limited amount of individual power supply in each node, it is imperative to design the energy-efficient distributed controller so as to meet the inherent energy constraints. The distributed eventtriggered control scheme could be a good candidate for the energy-saving purpose and, in [4], [6], [7], [9], [25], the distributed event-triggered controllers have been developed for multi-agent systems. Similarly, neural networks consist of a large number of neurons (or computing units in case of recurrent neural networks) and the state estimation of neural networks may consume large amounts of energy since each state of neurons should be estimated separately. In fact, in the implementation of a large-scale of artificial neural network, considerable processing and storage resources would have to be committed, and the corresponding resource allocation/saving becomes a critical issue. In this case, for the efficiency of energy utilization, it seems to be natural to introduce the event-triggering mechanism into the state estimation problem for neural networks. It should be pointed out that the event-triggered state estimation problem for neural networks has received very little research attention, and the corresponding research is still in its early stage.

In the theoretical modeling of traditional neural circuits [14], [34], each neuron is a simple analog processor and all neurons are connected by the synapses formed between neurons. In such a model, the system parameters are determined by the electric components such as capacitance and resistance. It is well known that the values of the capacitance and resistance are unstable that may be subject to unexpected random changes owing to the undesirable physical environ- ments such as high humidity and depression environment, surface oxidation between electrodes and leads, electrical and heat aging in dielectrics, etc. In other words, the network parameters (e.g., the capacitance and resistance) may exhibit unwanted fluctuations which may occur in a probabilistic way in terms of the factual situations where they are. This is particularly true for large-scale artificial neural networks where the system parameters are randomly fluctuated according to the network loads and communication constraints. Such kind of random changes of the network parameters may lead to some fundamental difficulties in dynamical analysis of the neural networks. For example, how can we establish a tractable model capable of describing the phenomenon of the random fluctuations as accurately as possible? How can we choose an appropriate stochastic analysis tool to deal with the random fluctuations of system parameters and thus derive the analysis results of the dynamics of the neural networks? It is, therefore, the second and primary motivation in our paper to provide satisfactory answers to the aforementioned two questions by designing an event-triggered state estimator for the multidelayed neural networks with the stochastic parameters.

In view of the above discussion, in this paper, the eventtriggered state estimation problem is addressed for a class of discrete-time multi-delayed neural networks with stochastic parameters and incomplete measurements. In the model of neural networks, we make the first attempt to introduce the stochastic parameters and study their effects on the dynamics of neural circuits. The delays among the interconnections are allowed to be different, which relaxes the assumptions in existing literature. The adopted measurement model is capable of representing randomly occurring sensor saturations and quantizations in a unified way. For the purpose of energy saving, the event-triggering mechanism is employed and an event-triggered state estimator is constructed for the neural networks under consideration. By using the Lyapunov-like theory, a sufficient condition is obtained under which the estimation error dynamics is exponentially ultimately bounded in the sense of mean square and the ultimate boundedness of the error dynamics can be estimated as well. Subsequently, the desired state estimator is designed in terms of the solution to a certain matrix inequality. Finally, a simulation example is utilized to demonstrate the effectiveness of the proposed event-triggered state estimation scheme.

Notation The notation used here is fairly standard except where otherwise stated. $\mathbb{R}^{n}$ denotes the $n$ dimensional Euclidean space. $\|A\|$ refers to the norm of a matrix $A$ defined by $\|A\|=\sqrt{\operatorname{trace}\left(A^{T} A\right)}$. The notation $X \geq Y$ (respectively, $X>Y$ ), where $X$ and $Y$ are real symmetric matrices, means that $X-Y$ is positive semi-definite (respectively, positive definite). $M^{T}$ represents the transpose of the matrix $M$. I denotes the identity matrix of compatible dimension. $\operatorname{diag}\{\ldots\}$ stands for a block-diagonal matrix and the notation $\operatorname{diag}_{n}\{\bullet\}$ is employed to stand for $\operatorname{diag}\{\overbrace{\bullet, \ldots, \bullet}^{n}\}$. E $\{x\}$ stands for the expectation of the stochastic variable $x$. Prob $\{\cdot\}$ means the occurrence probability of the event ".". o denotes the Hadamard product defined by $[A \circ B]_{i j}=A_{i j} B_{i j}$. In symmetric block matrices, " $*$ " is used as an ellipsis for terms 
induced by symmetry. Matrices, if they are not explicitly specified, are assumed to have compatible dimensions.

\section{Problem Formulation ANd Preliminaries}

Consider the following class of discrete-time multi-delayed neural networks with $n$ neurons

$$
\begin{aligned}
x_{i}(k+1)= & a_{i}(k) x_{i}(k)+\sum_{j=1}^{n} w_{i j}^{0} g_{j}\left(x_{j}(k)\right) \\
& +\sum_{j=1}^{n} w_{i j}^{1} g_{j}\left(x_{j}\left(k-\tau_{i j}\right)\right)+b_{i} \omega_{i}(k),
\end{aligned}
$$

for $i=1,2, \cdots, n$, where $x_{i}(k) \in \mathbb{R}$ is the state variable of neuron $i ; w_{i j}^{0}$ and $w_{i j}^{1}$ are the interconnection strength and the delayed interconnection strength between neurons $i$ and $j$, respectively; $\tau_{i j}$ is a constant representing the delay from neuron $i$ to $j ; g_{j}(\cdot)$ denotes the neuron activation function; $\omega_{i}(k)$ is a zero mean Gaussian white-noise process; and $b_{i}$ is a deterministic constant while $a_{i}(k)$ is a random variable satisfying $0<a_{i}(k)<1$.

Remark 1: The neural networks given by (1) is in nature a Hopfield neural network. Differently, we make the first attempt to introduce a set of stochastic variables $a_{i}(k)(i=$ $1,2, \cdots, n)$ to describe the fluctuations of the values of the capacitance and resistance. Moreover, the model of neural networks given by (1) admits different time delays for different interconnections. Such kind of multiple delays is more general than those in the existing literature.

Remark 2: In general, the model of neural network contains an external input which, in many neural network applications, is held constant over a time interval of interest [30]. By shifting the equilibrium point to the origin, the external input can be eliminated and, the estimate of the real state of neural networks can be obtained by re-shifting to the equilibrium point. In order to avoid unnecessary mathematical complexity, in this paper, we consider the neural networks without external inputs.

The neuron activation function $g_{i}$ satisfies $g_{i}(0)=0$ and the following Lipschitz condition:

$$
\left|g_{i}(x)-g_{i}(y)\right| \leq m_{i}|x-y|
$$

The random variables $\omega_{i}(k)$ and $a_{i}(k)$ have the following statistical properties

$$
\begin{aligned}
& \mathbb{E}\left\{a_{i}(k)\right\}=\bar{a}_{i}, \\
& \mathbb{E}\left\{a_{i}(k) a_{j}(k)\right\}=\tilde{a}_{i j}, \\
& \mathbb{E}\left\{\omega_{i}(k) \omega_{j}(k)\right\}=q_{i j},
\end{aligned}
$$

where $\bar{a}_{i}, \tilde{a}_{i j}$ and $q_{i j}$ are known constants. Moreover, $a_{i}(k)$ is assumed to be uncorrelated with the initial state $x_{i}(0)$ and the Gaussian white-noise noise $w_{i}(k)$.

$$
\begin{aligned}
& \text { Set } \\
& g(x(k))=\left[\begin{array}{llll}
g_{1}\left(x_{1}(k)\right) & g_{2}\left(x_{2}(k)\right) & \cdots & g_{n}\left(x_{n}(k)\right)
\end{array}\right]^{T}, \\
& x(k)=\left[\begin{array}{llll}
x_{1}(k) & x_{2}(k) & \cdots & x_{n}(k)
\end{array}\right]^{T}, \\
& w(k)=\left[\begin{array}{llll}
\omega_{1}(k) & \omega_{2}(k) & \cdots & \omega_{n}(k)
\end{array}\right]^{T}, \\
& A(k)=\operatorname{diag}\left\{a_{1}(k), a_{2}(k), \cdots, a_{n}(k)\right\} \\
& B=\operatorname{diag}\left\{\begin{array}{ll}
\left.b_{1}, b_{2}, \cdots, b_{n}\right\}, & W_{0}=\left[w_{i j}^{0}\right.
\end{array}\right]_{n \times n} \\
& E_{i}=\operatorname{diag}\{\underbrace{0, \cdots, 0}_{i-1}, 1 \underbrace{0, \cdots, 0}_{n-i}\}, \quad W_{1}=\left[w_{i j}^{1}\right]_{n \times n} .
\end{aligned}
$$

Then, the neural networks given by (1) can be written as the following compact form

$$
\begin{aligned}
x(k+1)= & A(k) x(k)+W_{0} g(x(k)) \\
& +\sum_{i=1}^{n} \sum_{j=1}^{n} E_{i} W_{1} E_{j} g\left(x\left(k-\tau_{i j}\right)\right)+B w(k) .
\end{aligned}
$$

In practice, the information about the neuron states is often incomplete from the network measurements and, meanwhile, the network measurements might be subject to the issues induced by limited communication. In this paper, both the quantization effects and sensor saturations are taken into account and the network measurement model is given as follows [33]:

$$
\begin{aligned}
y(k)= & \delta(\alpha(k), 1) C x(k)+\delta(\alpha(k), 2) s(C x(k)) \\
& +\delta(\alpha(k), 3) q(C x(k))+v(k)
\end{aligned}
$$

where $y(k) \in \mathbb{R}^{m}$ is the measurement output; $\delta(\cdot, \cdot)$ is the Kronecker delta function whose value is 1 if the two variables are equal, and 0 otherwise; $s(\cdot)$ is the saturation nonlinear function; $q(\cdot)$ is the quantization function; $C$ is a deterministic matrix with appropriate dimensions; $v(k) \in \mathbb{R}^{m}$ is the measurement noise which is a zero mean Gaussian white-noise process with $\mathbb{E}\left\{v(k) v^{T}(k)\right\}=Q_{v}$; and $\alpha(k)$ is a stochastic variable satisfying the following probability distribution:

$$
\operatorname{Prob}\{\alpha(k)=i\}=\beta_{i}, \quad i=1,2,3 .
$$

Here, $\alpha(k)$ is uncorrelated with other noise signals and $\beta_{i} \in$ $[0,1](i=1,2,3)$ are constants satisfying $\beta_{1}+\beta_{2}+\beta_{3}=1$.

The saturation function $s(\cdot)$ is defined by

$$
s(\vartheta)=\left[\begin{array}{llll}
s\left(\vartheta_{1}\right) & s\left(\vartheta_{2}\right) & \ldots & s\left(\vartheta_{m}\right)
\end{array}\right], \quad \forall \vartheta \in \mathbb{R}^{m}
$$

where $s\left(\vartheta_{i}\right)=\operatorname{sign}\left(\vartheta_{i}\right) \min \left\{\vartheta_{\max },\left|\vartheta_{i}\right|\right\}$ for each $i=$ $1,2, \ldots, m$, with $\vartheta_{\max }$ denoting the saturation level. The saturation function defined above is actually a nonlinear function and, for a given scalar $\tau$, we assume that

$$
[s(\tau)-\bar{k} \tau][s(\tau)-\tau] \leq 0
$$

where $\bar{k}$ is a positive scalar satisfying $0<\bar{k}<1$.

Remark 3: According to the definition of saturation function, it is easily seen that the saturation function $s(\cdot)$ satisfies the sector-bounded condition (9). The parameter $\bar{k}$ is used to characterize the lower bound of the sector-bounded nonlinearity and, in theory, parameter $\bar{k}$ should be taken as zero. 
However, this may be conservative. In practical applications, we usually choose the parameter $\bar{k}$ as a known scalar which can be determined by estimating the practical value of the measurement $y(k)$.

For the quantization function $q(\cdot)$, we adopt the logarithmictype quantizer defined by

$$
q(\vartheta)=\left[\begin{array}{llll}
q_{1}\left(\vartheta_{1}\right) & q_{2}\left(\vartheta_{2}\right) & \cdots & q_{m}\left(\vartheta_{m}\right)
\end{array}\right]^{T}, \quad \forall \vartheta \in \mathbb{R}^{m} .
$$

For each $q_{j}(\cdot)(1 \leq j \leq m)$, the set of quantization levels is described by

$$
\begin{gathered}
\mathcal{U}_{j}=\left\{ \pm u_{i}^{(j)}, u_{i}^{(j)}=\rho_{j}^{i} u_{0}^{(j)}, i=0, \pm 1, \pm 2, \cdots\right\} \cup\{0\} \\
0<\rho_{j}<1, \quad u_{0}^{(j)}>0,
\end{gathered}
$$

and $q_{j}(\cdot)$ is given by

$q_{j}\left(\vartheta_{j}\right)=\left\{\begin{array}{cc}u_{i}^{(j)}, & \frac{1}{1+\kappa_{j}} u_{i}^{(j)}<\vartheta_{j} \leq \frac{1}{1-\kappa_{j}} u_{i}^{(j)}, \\ 0, & \vartheta_{j}=0, \\ -q_{j}\left(-\vartheta_{j}\right), & \vartheta_{j} \leq 0\end{array}\right.$

with $\kappa_{j}=\left(1-\rho_{j}\right) /\left(1+\rho_{j}\right)$.

In this paper, we would like to estimate the neuron states by using the available network measurements given by (6). In order to save energy, we consider the event-triggered estimation scheme. Denote by $\left\{0=r_{0}<r_{1}<r_{2}<\cdots\right\}$ the sequence of event triggering instants determined by

$$
r_{l+1}=\min \left\{k \in \mathbb{N} \mid k>r_{l}, \xi^{T}(k) \xi(k)-\delta>0\right\}
$$

where $\xi(k)=y(k)-y\left(r_{l}\right)$ and $\delta$ is the triggering threshold.

The estimator structure adopted is given as follows:

$$
\begin{aligned}
\hat{x}(k+1)= & \bar{A} \hat{x}(k)+W_{0} g(\hat{x}(k)) \\
& +\sum_{i=1}^{n} \sum_{j=1}^{n} E_{i} W_{1} E_{j} g\left(\hat{x}\left(k-\tau_{i j}\right)\right) \\
& +G\left(y\left(r_{l}\right)-C \hat{x}(k)\right),
\end{aligned}
$$

for $k \in\left[r_{l}, r_{l+1}\right)$, where $\bar{A}=\operatorname{diag}\left\{\bar{a}_{1}, \bar{a}_{2}, \cdots, \bar{a}_{n}\right\}$ and $G$ is the estimator gain to be designed.

Remark 4: The Hopfield neural network is actually a modeling of neural circuits where each neuron is a simple analog processor, while the rich connectivity provided in real neural circuits by the synapses formed between neurons are provided by the parallel communication lines in the valuepassing analog processor networks [34]. This kind of neural networks happen to fall into the category of the artificial neural networks mentioned in this paper, and the energy-saving problem seems to be important when the neurons' states are estimated. Therefore, the event-triggered estimation scheme could be a good candidate for the energy-saving purpose.
By letting the estimation error be $e(k)=x(k)-\hat{x}(k)$, it follows from (5) and (10) that

$$
\begin{aligned}
e(k+1)= & \tilde{A}(k) x(k)+\left(1-\beta_{1}\right) G C x(k) \\
& +(\bar{A}-G C) e(k)+W_{0} \tilde{g}(k)+G \xi(k) \\
& +\bar{B} \bar{w}(k)+\sum_{i=1}^{n} \sum_{j=1}^{n} E_{i} W_{1} E_{j} \tilde{g}\left(k-\tau_{i j}\right) \\
& -\beta_{2} G s(C x(k))-\beta_{3} G q(C x(k)) \\
& -\tilde{\delta}_{1}^{\alpha}(k) G C x(k)-\tilde{\delta}_{2}^{\alpha}(k) G s(C x(k)) \\
& -\tilde{\delta}_{3}^{\alpha}(k) G q(C x(k)), \quad k \in\left[r_{l}, r_{l+1}\right),
\end{aligned}
$$

where

$$
\begin{aligned}
& \tilde{A}(k)=A(k)-\bar{A}, \quad \tilde{g}(k)=g(x(k))-g(\hat{x}(k)), \\
& \bar{B}=\left[\begin{array}{ll}
B & -G
\end{array}\right], \quad \bar{w}(k)=\left[\begin{array}{ll}
w^{T}(k) & v^{T}(k)
\end{array}\right]^{T}, \\
& \tilde{\delta}_{i}^{\alpha}(k)=\delta(\alpha(k), i)-\beta_{i}, \quad i=1,2,3 .
\end{aligned}
$$

Furthermore, set $\eta(k)=\left[\begin{array}{ll}x^{T}(k) & e^{T}(k)\end{array}\right]^{T}$. Then, the dynamics of the neural network (5) and the error system (11) can be expressed by the following augmented system

$$
\begin{aligned}
\eta(k+1)= & \mathcal{A} \eta(k)+\bar{W}_{0} \mathcal{G}(k)+\sum_{i=1}^{n} \sum_{j=1}^{n} \bar{W}_{i j}^{1} \mathcal{G}\left(k-\tau_{i j}\right) \\
& +\mathcal{B} \bar{w}(k)+H_{1} G \xi(k)+\tilde{\mathcal{A}}(k) H_{2} \eta(k) \\
& -\beta_{1} H_{1} G C H_{2} \eta(k) \\
& -\beta_{2} H_{1} G s\left(C H_{2} \eta(k)\right) \\
& -\beta_{3} H_{1} G q\left(C H_{2} \eta(k)\right) \\
& -\tilde{\delta}_{1}^{\alpha}(k) H_{1} G C H_{2} \eta(k) \\
& -\tilde{\delta}_{2}^{\alpha}(k) H_{1} G s\left(C H_{2} \eta(k)\right) \\
& -\tilde{\delta}_{3}^{\alpha}(k) H_{1} G q\left(C H_{2} \eta(k)\right)
\end{aligned}
$$

where

$$
\begin{aligned}
& \mathcal{A}=\left[\begin{array}{cc}
\bar{A} & 0 \\
G C & \bar{A}-G C
\end{array}\right], \quad H_{1}=\left[\begin{array}{l}
0 \\
I
\end{array}\right], \\
& \bar{W}_{0}=\operatorname{diag}_{2}\left\{W_{0}\right\}, \quad \bar{W}_{i j}^{1}=\operatorname{diag}_{2}\left\{E_{i} W_{1} E_{j}\right\}, \\
& \mathcal{B}=\left[\begin{array}{cc}
B & 0 \\
B & -G
\end{array}\right], \quad \tilde{\mathcal{A}}(k)=\left[\begin{array}{c}
\tilde{A}(k) \\
\tilde{A}(k)
\end{array}\right], \\
& \mathcal{G}(k)=\left[\begin{array}{c}
g(x(k)) \\
\tilde{g}(k)
\end{array}\right], \quad H_{2}=\left[\begin{array}{ll}
I & 0
\end{array}\right] .
\end{aligned}
$$

Definition 1: [37] The dynamics of the augmented system (13) is exponentially ultimately bounded in the mean square if there exist constants $0<\mu<1, \nu>0, \bar{\kappa}>0$ such that

$$
\mathbb{E}\left\{\|\eta(k)\|^{2}\right\} \leq \mu^{k} \nu+\kappa(k), \text { and } \lim _{k \rightarrow+\infty} \kappa(k)=\bar{\kappa} .
$$

The aim of this paper is to design an event-triggered estimator with the form (10) for the multi-delayed neural networks (1) with incomplete measurements described by (6). More specifically, we are interested in looking for the estimator parameter $G$ such that the dynamics of the augmented system (13) is exponentially mean-square ultimately bounded with a satisfactory bound. 


\section{Main Results}

In this section, the boundedness issue is first analyzed for the augmented system (13). Then, according to the conducted analysis results, we shall investigate the design problem of the state estimator and give the desired estimator gain in terms of the solution to a certain matrix inequality.

For the logarithmic-type quantization function $q(\cdot)$, it is shown in [11] that $q_{j}\left(\vartheta_{j}\right)=\left(1+\Delta_{j}\right) \vartheta_{j}$ such that $\left|\Delta_{j}\right| \leq \kappa_{j}$. Setting $\Delta=\operatorname{diag}\left\{\Delta_{1}, \ldots, \Delta_{m}\right\}, \Lambda_{q}=\operatorname{diag}\left\{\kappa_{1}, \ldots, \kappa_{m}\right\}$ and $F=\Delta \Lambda_{q}^{-1}$, the quantization effect can be described as

$$
q\left(\mathrm{CH}_{2} \eta(k)\right)=U \eta(k)
$$

where $U=\left(I+F \Lambda_{q}\right) C H_{2}$ and $F F^{T}=F^{T} F \leq I$.

For the purpose of the notation simplicity, we denote $s\left(\mathrm{CH}_{2} \eta(k)\right)$ by $\bar{s}(k)$ and set

$$
\begin{aligned}
& \overline{\mathcal{G}}_{i}(k)=\left[\begin{array}{llll}
\mathcal{G}^{T}\left(k-\tau_{i 1}\right) & \mathcal{G}^{T}\left(k-\tau_{i 2}\right) & \cdots & \mathcal{G}^{T}\left(k-\tau_{i n}\right)
\end{array}\right]^{T}, \\
& \overline{\mathcal{G}}(k)=\left[\begin{array}{llll}
\overline{\mathcal{G}}_{1}^{T}(k) & \overline{\mathcal{G}}_{2}^{T}(k) & \cdots & \overline{\mathcal{G}}_{n}^{T}(k)
\end{array}\right]^{T}, \\
& \bar{W}_{i}^{1}=\left[\begin{array}{llll}
\bar{W}_{i 1}^{1} & \bar{W}_{i 2}^{1} & \cdots & \bar{W}_{i n}^{1}
\end{array}\right], \\
& \bar{W}_{1}=\left[\begin{array}{llll}
\bar{W}_{1}^{1} & \bar{W}_{2}^{1} & \cdots & \bar{W}_{n}^{1}
\end{array}\right] .
\end{aligned}
$$

Then, the augmented system given by (13) can be rewritten as the following concise form:

$$
\begin{aligned}
\eta(k+1)= & \mathcal{A} \eta(k)+\bar{W}_{0} \mathcal{G}(k)+\bar{W}_{1} \overline{\mathcal{G}}(k)+\mathcal{B} \bar{w}(k) \\
& +H_{1} G \xi(k)+\tilde{\mathcal{A}}(k) H_{2} \eta(k) \\
& -\beta_{1} H_{1} G C H_{2} \eta(k)-\beta_{2} H_{1} G \bar{s}(k) \\
& -\beta_{3} H_{1} G U \eta(k)-\tilde{\delta}_{1}^{\alpha}(k) H_{1} G C H_{2} \eta(k) \\
& -\tilde{\delta}_{2}^{\alpha}(k) H_{1} G \bar{s}(k)-\tilde{\delta}_{3}^{\alpha}(k) H_{1} G U \eta(k) .
\end{aligned}
$$

The following lemma will be used in the proof of our main results.

Lemma 1: Under the condition (2), we have

$$
\mathcal{G}^{T}(k) \mathcal{G}(k)-\eta^{T}(k) \bar{M} \eta(k) \leq 0
$$

where $\bar{M}=\operatorname{diag}_{2}\{M\}$ and $M=\operatorname{diag}\left\{m_{1}^{2}, m_{2}^{2}, \cdots, m_{n}^{2}\right\}$.

Proof: From the definitions of $e(k)$ and $\eta(k)$ together with (4), (12) and (14), it can be obtained that

$$
\begin{aligned}
& \mathcal{G}^{T}(k) \mathcal{G}(k)-\eta^{T}(k) \bar{M} \eta(k) \\
= & g^{T}(x(k)) g(x(k))-x^{T}(k) M x(k) \\
& +\tilde{g}^{T}(k) \tilde{g}(k)-e^{T}(k) M e(k) \\
= & \sum_{i=1}^{n}\left(g_{i}^{2}\left(x_{i}(k)\right)-m_{i}^{2} x_{i}^{2}(k)\right) \\
& +\sum_{i=1}^{n}\left(\left(g_{i}\left(x_{i}(k)\right)-g_{i}\left(\hat{x}_{i}(k)\right)\right)^{2}\right. \\
& \left.-m_{i}^{2}\left(x_{i}(k)-\hat{x}_{i}(k)\right)^{2}\right) .
\end{aligned}
$$

It is easily seen from (2) that $g_{i}^{2}\left(x_{i}(k)\right)-m_{i}^{2} x_{i}^{2}(k)<0$ and $\left(g_{i}\left(x_{i}(k)\right)-g_{i}\left(\hat{x}_{i}(k)\right)\right)^{2}-m_{i}^{2}\left(x_{i}(k)-\hat{x}_{i}(k)\right)^{2}<0$, from which the inequality (18) follows directly. Therefore, the proof of this lemma is complete.

Setting $\hat{M}=\operatorname{diag}_{n^{2}}\{\bar{M}\}$, we further have

$$
\overline{\mathcal{G}}^{T}(k) \overline{\mathcal{G}}(k)-\eta_{d}^{T}(k) \hat{M} \eta_{d}(k) \leq 0
$$

where $\eta_{d}(k)=\left[\begin{array}{llll}\bar{\eta}_{1}^{T}(k) & \bar{\eta}_{2}^{T}(k) & \cdots & \bar{\eta}_{n}^{T}(k)\end{array}\right]^{T}$ and $\bar{\eta}_{i}(k)=\left[\begin{array}{llll}\eta^{T}\left(k-\tau_{i 1}\right) & \eta^{T}\left(k-\tau_{i 2}\right) & \cdots & \eta^{T}\left(k-\tau_{i n}\right)\end{array}\right]^{T}$.

Lemma 2: The saturation function $\bar{s}(k)$ satisfies

$$
\begin{array}{r}
\bar{s}^{T}(k) \bar{s}(k)-\eta^{T}(k) H_{2}^{T} C^{T}\left(K^{T}+I\right) \bar{s}(k) \\
+\eta^{T}(k) H_{2}^{T} C^{T} K^{T} C H_{2} \eta(k) \leq 0
\end{array}
$$

where $K=\operatorname{diag}_{m}\{\bar{k}\}$.

Proof: According to (9), we have

$$
\begin{aligned}
& (s(\vartheta)-K \vartheta)^{T}(s(\vartheta)-\vartheta) \\
= & \sum_{i=1}^{m}\left(\left(s\left(\vartheta_{i}\right)-\bar{k} \vartheta_{i}\right)^{T}\left(s\left(\vartheta_{i}\right)-\vartheta_{i}\right)\right) \\
\leq & 0 .
\end{aligned}
$$

Letting $\vartheta$ be $\vartheta=C_{2} \eta(k)$ and noting $\bar{s}(k)=$ $s\left(\mathrm{CH}_{2} \eta(k)\right)$, it immediately follows from the above inequality that

$$
\left(\bar{s}(k)-K_{C} H_{2} \eta(k)\right)^{T}\left(\bar{s}(k)-C_{2} \eta(k)\right) \leq 0
$$

which is the exactly same as inequality (20) and hence the proof of this lemma is accomplished.

Lemma 3: [13] For a stochastic variable $\alpha(k)$ satisfying the probability distribution given by (7), we have

$$
\mathbb{E}\left\{\tilde{\delta}_{i}^{\alpha}(k) \tilde{\delta}_{j}^{\alpha}(k)\right\}= \begin{cases}\beta_{i}\left(1-\beta_{i}\right), & i=j, \\ -\beta_{i} \beta_{j}, & i \neq j .\end{cases}
$$

for $i, j=1,2,3$.

Lemma 4: Let $T, N$ and $F$ be real matrices of appropriate dimensions with $F$ satisfying $F^{T} F \leq I$. Then, for any scalar $\epsilon>0$, we have

$$
T F N+(T F N)^{T} \leq \epsilon^{-1} T T^{T}+\epsilon N^{T} N .
$$

In the following theorem, a sufficient condition is provided under which the augmented system (13) is exponentially ultimately bounded in the mean square.

Theorem 1: For the given estimator parameter $G$, the augmented system (13) is exponentially ultimately bounded in the mean square if there exist positive definite matrices $P=$ $\left[P_{i j}\right]_{2 \times 2}, Q_{i j}(i, j=1,2, \cdots, n)$ and positive scalars $\lambda_{1}, \lambda_{2}$, $\lambda_{3}, \lambda_{4}$ satisfying the following inequality:

$$
\Phi=\left[\begin{array}{cccccc}
\Theta_{11} & \Theta_{12} & \Theta_{13} & \Theta_{14} & \Theta_{15} & 0 \\
* & \Theta_{22} & \Theta_{23} & \Theta_{24} & \Theta_{25} & 0 \\
* & * & \Theta_{33} & \Theta_{34} & \Theta_{35} & 0 \\
* & * & * & \Theta_{44} & 0 & 0 \\
* & * & * & * & \Theta_{55} & 0 \\
* & * & * & * & * & \Theta_{66}
\end{array}\right]<0
$$

where

$$
\begin{aligned}
\Theta_{11}= & \left(1+\beta_{2}+\beta_{3}\right) \mathcal{A}^{T} P \mathcal{A}+H_{2}^{T} \tilde{P} H_{2} \\
& +5 \beta_{3} U^{T} G^{T} H_{1}^{T} P H_{1} G U \\
& +\beta_{1} H_{2}^{T} C^{T} G^{T} H_{1}^{T} P H_{1} G C H_{2} \\
& -\beta_{1} \mathcal{A}^{T} P H_{1} G C H_{2} \\
& -\beta_{1} H_{2}^{T} C^{T} G^{T} H_{1}^{T} P \mathcal{A}-P+\lambda_{1} \bar{M} \\
& +\sum_{i=1}^{n} \sum_{j=1}^{n} Q_{i j}-\lambda_{3} H_{2}^{T} C^{T} K^{T} C H_{2},
\end{aligned}
$$




$$
\begin{aligned}
\Theta_{12} & =\mathcal{A}^{T} P \bar{W}_{0}-\beta_{1} H_{2}^{T} C^{T} G^{T} H_{1}^{T} P \bar{W}_{0}, \\
\Theta_{13} & =\mathcal{A}^{T} P \bar{W}_{1}-\beta_{1} H_{2}^{T} C^{T} G^{T} H_{1}^{T} P \bar{W}_{1}, \\
\Theta_{14} & =\mathcal{A}^{T} P H_{1} G-\beta_{1} H_{2}^{T} C^{T} G^{T} H_{1}^{T} P H_{1} G, \\
\Theta_{15} & =\frac{1}{2} \lambda_{3} H_{2}^{T} C^{T}\left(K^{T}+I\right), \\
\Theta_{22} & =\left(1+\beta_{3}\right) \bar{W}_{0}^{T} P \bar{W}_{0}-\lambda_{1} I, \\
\Theta_{23} & =\bar{W}_{0}^{T} P \bar{W}_{1}, \quad \Theta_{24}=\bar{W}_{0}^{T} P H_{1} G, \\
\Theta_{25} & =-\beta_{2} \bar{W}_{0}^{T} P H_{1} G, \\
\Theta_{33} & =\left(1+\beta_{3}\right) \bar{W}_{1}^{T} P \bar{W}_{1}-\lambda_{2} I, \\
\Theta_{34} & =\bar{W}_{1}^{T} P H_{1} G, \quad \Theta_{35}=-\beta_{2} \bar{W}_{1}^{T} P H_{1} G, \\
\Theta_{44} & =\left(1+\beta_{2}+\beta_{3}\right) G^{T} H_{1}^{T} P H_{1} G-\lambda_{4} I, \\
\Theta_{55} & =3 \beta_{2} G^{T} H_{1}^{T} P H_{1} G-\lambda_{3} I, \\
\Theta_{66} & =-Q+\lambda_{2} \hat{M}, \quad E=\left[\tilde{a}_{i j}-\bar{a}_{i} \bar{a}_{j}\right]_{n \times n}, \\
Q_{i} & =\operatorname{diag}\left\{Q_{i 1}, Q_{i 2}, \cdots, Q_{i n}\right\}, \\
Q & =\operatorname{diag}\left\{Q_{1}, Q_{2}, \cdots, Q_{n}\right\}, \\
\tilde{P} & =E \circ\left(P_{11}+P_{12}+P_{21}+P_{22}\right) .
\end{aligned}
$$

Furthermore, if the inequality (22) is solvable, the ultimate bound of the dynamics of the augmented system (13) is given by

$$
\bar{\kappa}=\frac{\alpha_{0}}{\left(\alpha_{0}-1\right) \lambda_{\min }(P)}\left(\lambda_{4} \delta+\vartheta\right)
$$

where $\vartheta=\lambda_{\max }\left(\mathcal{B}^{T} P \mathcal{B}\right)\left(\sum_{i=1}^{n} q_{i i}+\operatorname{trace}\left(Q_{v}\right)\right)$ and $\alpha_{0}>1$ satisfies

$$
\begin{aligned}
\phi\left(\alpha_{0}\right)+\frac{1}{\alpha_{0}-1} \sum_{i=1}^{n} \sum_{j=1}^{n} \varphi_{i j}\left(\alpha_{0}\right) & \\
& \times\left(\alpha_{0}^{\tau_{i j}+1}+\alpha_{0}^{\tau_{i j}}-2 \alpha_{0}\right)=0
\end{aligned}
$$

with

$$
\begin{aligned}
& \phi\left(\alpha_{0}\right)=\left(\alpha_{0}-1\right) \lambda_{\max }(P)-\alpha_{0} \lambda_{\min }(-\Phi), \\
& \varphi_{i j}\left(\alpha_{0}\right)=\left(\alpha_{0}-1\right) \lambda_{\max }\left(Q_{i j}\right) .
\end{aligned}
$$

Proof: See Appendix I.

Remark 5: In Theorem 1, a sufficient condition is provided under which the dynamics of the augmented system (13) is exponentially mean-square ultimately bounded and the ultimate bound of the error dynamics is given. It can be seen from (23) that the ultimate bound is dependent on both the eventtriggering threshold and the variances of external/measurement noises. Such a bound can be reduced by decreasing the eventtriggering threshold or depressing the noises' variances. Note that the ultimate boundedness is also related to other matrix parameters.

Remark 6: The measurement model (6) has been firstly proposed in [33]. In the proof of the main results of [33], the last three terms of (17) were assumed to be unrelated, which is actually not the case. Fortunately, by using Lemma 3 and following the lines in the proof of Theorem 1 in this paper, the main results in [33] can be easily rectified.

By means of Theorem 1, the design problem of the desired estimator shall be investigated. For the convenience of design, the positive definite matrix $P$ is taken as $P=\operatorname{diag}\left\{P_{11}, P_{22}\right\}$ where $P_{11}$ and $P_{22}$ are positive definite matrices.
In the following theorem, the design method of the desired estimator gain is given in terms of the solution to a linear matrix inequality.

Theorem 2: Consider the stochastic neural network given by (1) with the incomplete measurements described by (6). If there exist positive definite matrices $P=\operatorname{diag}\left\{P_{11}, P_{22}\right\}, Q_{i j}$ $(i, j=1,2, \cdots, n)$, matrix $Y$ and positive scalars $\lambda_{1}, \lambda_{2}, \lambda_{3}$, $\lambda_{4}, \epsilon$ satisfying the following inequality:

$$
\begin{aligned}
& \Upsilon= \\
& {\left[\begin{array}{cccccccccc}
\Xi_{11} & 0 & \Xi_{13} & 0 & \Xi_{15} & \Xi_{16} & \Xi_{17} & 0 & 0 & 0 \\
* & \Xi_{22} & \Xi_{23} & 0 & \Xi_{25} & 0 & 0 & \Xi_{28} & 0 & 0 \\
* & * & \Xi_{33} & 0 & 0 & 0 & 0 & 0 & \Xi_{39} & 0 \\
* & * & * & \Xi_{44} & 0 & 0 & 0 & 0 & 0 & 0 \\
* & * & * & * & \Xi_{55} & 0 & 0 & 0 & 0 & 0 \\
* & * & * & * & * & \Xi_{66} & 0 & 0 & 0 & 0 \\
* & * & * & * & * & * & \Xi_{77} & 0 & 0 & \Xi_{70} \\
* & * & * & * & * & * & * & \Xi_{88} & 0 & 0 \\
* & * & * & * & * & * & * & * & \Xi_{99} & 0 \\
* & * & * & * & * & * & * & * & * & \Xi_{00}
\end{array}\right]}
\end{aligned}
$$$$
<0
$$

where

$$
\begin{aligned}
\Xi_{11}= & H_{2}^{T} \tilde{P} H_{2}-P+\lambda_{1} \bar{M}+\sum_{i=1}^{n} \sum_{j=1}^{n} Q_{i j} \\
& -\lambda_{3} H_{2}^{T} C^{T} K C H_{2}+\epsilon H_{2}^{T} C^{T} \Lambda_{q}^{2} C H_{2}, \\
\Xi_{13}= & \frac{1}{2} \lambda_{3} H_{2}^{T} C^{T}\left(K^{T}+I\right), \quad \Xi_{15}=\mathcal{A}_{Y}^{T}-\beta_{1} H_{2}^{T} C^{T} G_{Y}^{T}, \\
\Xi_{16}= & {\left[\begin{array}{ll}
\sqrt{\beta_{2}+\beta_{3}} \mathcal{A}_{Y}^{T} & \sqrt{\beta_{1}\left(1-\beta_{1}\right)} H_{2}^{T} C^{T} G_{Y}^{T}
\end{array}\right], } \\
\Xi_{17}= & \sqrt{5 \beta_{3}} H_{2}^{T} C^{T} G_{Y}^{T}, \\
\Xi_{23}= & {\left[\begin{array}{ll}
-\beta_{2} G_{Y}^{T} \bar{W}_{0} & -\beta_{2} G_{Y}^{T} \bar{W}_{1} \quad 0
\end{array}\right]^{T}, } \\
\Xi_{25}= & {\left[\begin{array}{lll}
P \bar{W}_{0} & P \bar{W}_{1} & G_{Y}
\end{array}\right]^{T}, } \\
\Xi_{28}= & {\left[\begin{array}{lll}
0 & 0 & \sqrt{\beta_{2}+\beta_{3}} G_{Y}
\end{array}\right]^{T}, } \\
\Xi_{22}= & \operatorname{diag}\left\{\beta_{3} \bar{W}_{0}^{T} P \bar{W}_{0}-\lambda_{1} I, \beta_{3} \bar{W}_{1}^{T} P \bar{W}_{1}-\lambda_{2} I,-\lambda_{4} I\right\}, \\
\Xi_{33}= & -\lambda_{3} I, \quad \Xi_{39}=\sqrt{3 \beta_{2}} G_{Y}^{T}, \quad \Xi_{44}=-Q+\lambda_{2} \hat{M}, \\
\Xi_{55}= & \Xi_{77}=\Xi_{88}=\Xi_{99}=-P, \quad \Xi_{66}=\operatorname{diag}_{2}\{-P\}, \\
\Xi_{70}= & \sqrt{5 \beta_{3}} G_{Y}, \quad \Xi_{00}=-\epsilon I, \quad G_{Y}=\left[\begin{array}{ll}
0 & Y^{T}
\end{array}\right]^{T}, \\
\mathcal{A}_{Y}= & {\left[\begin{array}{ll}
P_{11} \bar{A} & 0 \\
Y C & P_{22} \bar{A}-Y C
\end{array}\right], }
\end{aligned}
$$

then, the design problem of the desired state estimator (10) is solvable. Furthermore, if the inequality (26) is feasible, the desired state estimator gain is given by

$$
G=P_{22}^{-1} Y \text {. }
$$

Proof: See Appendix II.

Remark 7: In this paper, the event-triggered state estimation problem is investigated for a class of multi-delayed neural networks with stochastic parameters and incomplete measurements. The main novelty can be summarized as follows: 1) the stochastic parameters are introduce for the first time to account for the fluctuations of the capacitance and resistance in the neural circuits, 2) different time delays for different interconnections are taken into account which extends ones in the existing literature, and 3) the event-triggering mechanism is employed to estimate the neuron's states of multi-delayed neural networks with stochastic parameters. Moreover, we 
adopt a unified measurement model which is capable of reflecting randomly occurring sensor saturations and randomly occurring quantizations. Finally, the state estimator is designed such that, for all admissible incomplete measurements as well as the stochastic parameters, the estimation error dynamics is exponentially mean-square ultimately bounded. In the next section, a simulation example is given to illustrative the effectiveness of the proposed state estimation scheme.

\section{AN ILLUSTRATIVE EXAMPLE}

In this section, a numerical simulation example is presented to demonstrate the effectiveness of the proposed eventtriggered state estimation scheme for the multi-delayed neural networks with the stochastic parameters, randomly occurring sensor saturations as well as randomly occurring quantizations.

Consider a class of discrete-time multi-delayed neural networks described by (1) with three neurons. The deterministic system parameters are chosen as $b_{1}=b_{3}=0.2$ and $b_{2}=0.1$ and the expectations of the stochastic parameters are set as $\bar{a}_{1}=0.2, \bar{a}_{2}=0.3$ and $\bar{a}_{3}=0.4$. The delays among the interconnections are taken as $\tau_{11}=\tau_{13}=\tau_{22}=\tau_{23}=$ $\tau_{31}=\tau_{32}=\tau_{33}=1$ and $\tau_{12}=\tau_{21}=2$. The coupled configuration matrix and the delayed coupled configuration matrix are, respectively, given by

$W_{0}=\left[\begin{array}{ccc}-0.3 & 0.1 & 0.2 \\ 0.1 & -0.2 & 0.1 \\ 0.2 & 0.1 & -0.3\end{array}\right], W_{1}=\left[\begin{array}{ccc}-0.2 & 0.1 & 0.1 \\ 0.1 & -0.2 & 0.1 \\ 0.1 & 0.1 & -0.2\end{array}\right]$

The neuron activation functions are assumed to be of the following form:

$$
\begin{aligned}
& g_{1}\left(x_{1}(k)\right)=\tanh \left(0.2 x_{1}(k)\right), \\
& g_{2}\left(x_{2}(k)\right)=\tanh \left(-0.15 x_{2}(k)\right), \\
& g_{3}\left(x_{3}(k)\right)=\tanh \left(0.23 x_{3}(k)\right)
\end{aligned}
$$

which satisfy (2) with

$$
m_{1}=0.2, \quad m_{2}=0.15, \quad m_{3}=0.23 .
$$

In the measurement model described by (6), the parameter matrix $C$ is taken as $C=\left[\begin{array}{lll}0.5 & -0.3 & 0.4\end{array}\right]$, the parameters of saturation function are selected as $k=0.4$ and $\vartheta_{\max }=0.05$, the quantizer parameters are set as $\kappa=0.25$ and $u_{0}^{(1)}=3$, and the probabilities are given by $\beta_{1}=0.5, \beta_{2}=0.2$ and $\beta_{3}=0.3$.

In this example, the bound of the triggering condition is chosen as $\delta=1$. With the above parameters, by using the Matlab with LMI Toolbox, we solve the matrix inequality (26) and obtain a feasible solution as follows (only the main parameters are listed):

$$
P_{22}=\left[\begin{array}{lll}
4.4917 & 0.0902 & 0.8268 \\
0.0902 & 4.8111 & 0.1857 \\
0.8268 & 0.1857 & 4.0158
\end{array}\right], \quad Y=\left[\begin{array}{c}
0.0759 \\
-0.0530 \\
0.0786
\end{array}\right] .
$$

Then, according to (27), the desired estimator gain is designed as

$$
G=\left[\begin{array}{c}
0.0140 \\
-0.0120 \\
0.0173
\end{array}\right]
$$
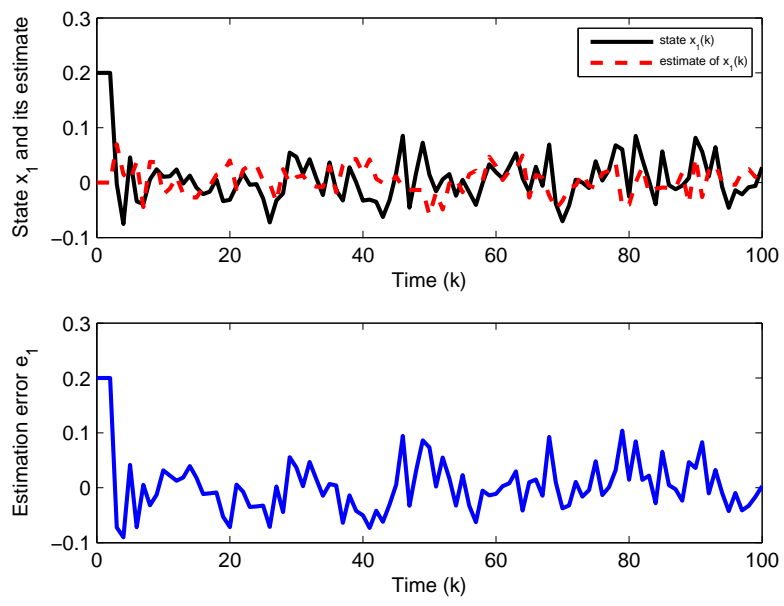

Fig. 1. State $x_{1}(k)$, its estimate and estimation error.
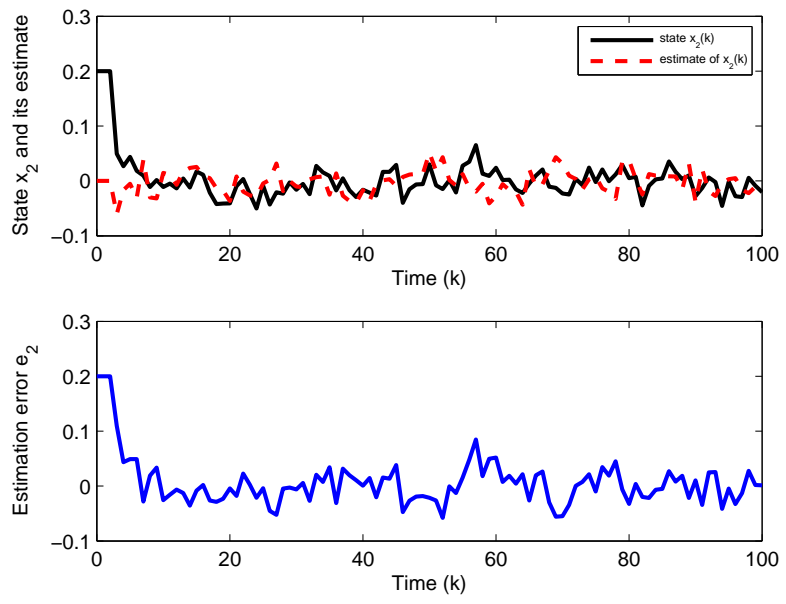

Fig. 2. State $x_{2}(k)$, its estimate and estimation error.

In the simulation, the initial values of the states are taken as $x_{1}(s)=0.2, x_{2}(s)=0.2$ and $x_{3}(s)=-0.3$ for $s \in$ $[0,2]$ and the variances of Gaussian white-noises are set as $q_{11}=q_{22}=0.2, q_{33}=0.3, q_{i j}=0(i \neq j)$ and $Q_{v}=$ 2. Simulation results are shown in Figs. 1-4. In Fig. 1, the above picture plots the real state $x_{1}(k)$ and its estimate $\hat{x}_{1}(k)$ and the state estimation error $e_{1}(k)$ is depicted in the picture below. Same results are shown in Figs. 2 and 3 for states $x_{2}(k)$ and $x_{3}(k)$, respectively. Fig. 4 draws the event-based release instants and the corresponding release intervals. The simulation results have demonstrated that the designed state estimator performs very well.

\section{CONCLUSiOnS}

In this paper, we have investigated the event-triggered state estimation problem for a class of discrete-time multi-delayed neural networks with stochastic parameters and incomplete measurements. In the model of neural networks, a set of stochastic variables has been introduced to characterize the random fluctuations of the system parameters and the delays among the interconnections have been assumed to be different 

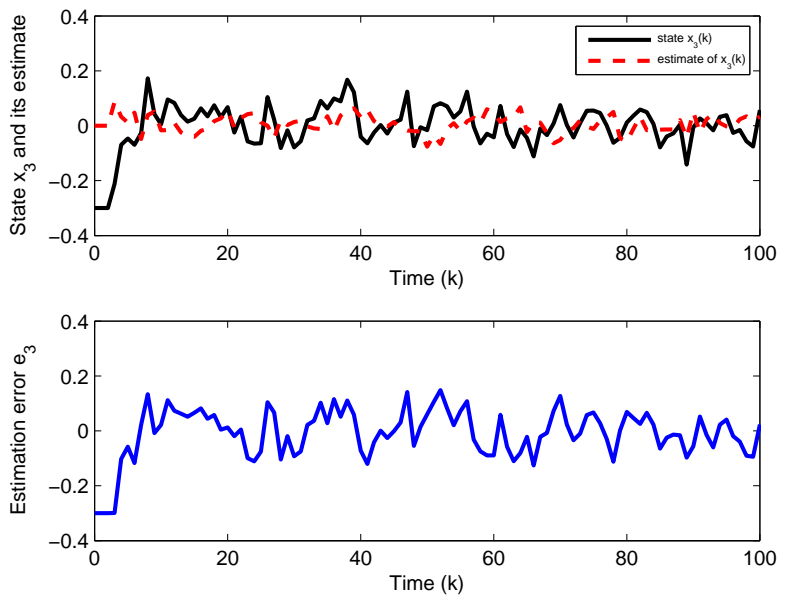

Fig. 3. State $x_{3}(k)$, its estimate and estimation error

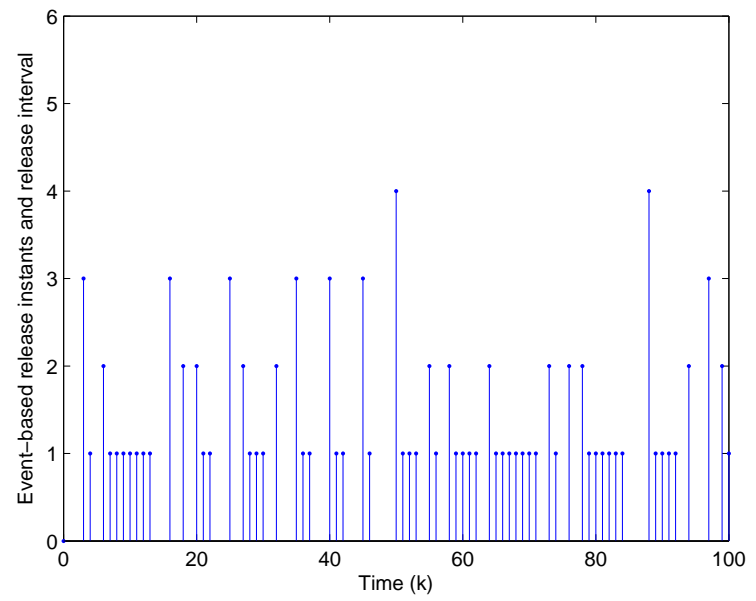

Fig. 4. Event-based release instants and release intervals

in order to cater to more realistic neural networks. Based on an unified measurement model that describes randomly occurring sensor saturations and quantizations, an event-triggered state estimator has been constructed. By using the Lyapunov-like theory, the desired state estimator has been designed such that the estimation error dynamics is exponentially ultimately bounded in the mean square and the ultimate boundedness of the error dynamics has been given. Finally, the effectiveness of the proposed event-triggered state estimation approach has been demonstrated by a simulation example. Future research topics include the extension of the results to the continuoustime delayed neural networks (see e.g. [22], [28]) with incomplete information.

\section{APPENDIX I}

\section{ProOF OF THEOREM 1}

Proof: In this proof, the exponentially mean-square ultimate boundedness of the augmented system (13) is shown with the help of Lyapynov functional-like theory.
Define the following functional

$$
V(k)=\eta^{T}(k) P \eta(k)+\sum_{i=1}^{n} \sum_{j=1}^{n} \sum_{l=k-\tau_{i j}}^{k-1} \eta^{T}(l) Q_{i j} \eta(l)
$$

and calculate the difference of $V(k)$ along the system (17) as follows:

$$
\begin{aligned}
& \mathbb{E}\{V(k+1)-V(k)\} \\
& =\mathbb{E}\left\{\eta^{T}(k) \mathcal{A}^{T} P \mathcal{A} \eta(k)+\mathcal{G}^{T}(k) \bar{W}_{0}^{T} P \bar{W}_{0} \mathcal{G}(k)\right. \\
& +\overline{\mathcal{G}}^{T}(k) \bar{W}_{1}^{T} P \bar{W}_{1} \overline{\mathcal{G}}(k)+\xi^{T}(k) G^{T} H_{1}^{T} P H_{1} G \xi(k) \\
& +\eta^{T}(k) H_{2}^{T} \tilde{\mathcal{A}}^{T}(k) P \tilde{\mathcal{A}}(k) H_{2} \eta(k) \\
& +\beta_{1}^{2} \eta^{T}(k) H_{2}^{T} C^{T} G^{T} H_{1}^{T} P H_{1} G C H_{2} \eta(k) \\
& +\beta_{2}^{2} \bar{s}^{T}(k) G^{T} H_{1}^{T} P H_{1} G \bar{s}(k)+\bar{w}^{T}(k) \mathcal{B}^{T} P \mathcal{B} \bar{w}(k) \\
& +\beta_{3}^{2} \eta^{T}(k) U^{T} G^{T} H_{1}^{T} P H_{1} G U \eta(k) \\
& +\tilde{\delta}_{1}^{\alpha 2}(k) \eta^{T}(k) H_{2}^{T} C^{T} G^{T} H_{1}^{T} P H_{1} G C H_{2} \eta(k) \\
& +\tilde{\delta}_{2}^{\alpha 2}(k) \bar{s}^{T}(k) G^{T} H_{1}^{T} P H_{1} G \bar{s}(k) \\
& +\tilde{\delta}_{3}^{\alpha 2}(k) \eta^{T}(k) U^{T} G^{T} H_{1}^{T} P H_{1} G U \eta(k) \\
& +2 \eta^{T}(k) \mathcal{A}^{T} P \bar{W}_{0} \mathcal{G}(k)+2 \eta^{T}(k) \mathcal{A}^{T} P \bar{W}_{1} \overline{\mathcal{G}}(k) \\
& +2 \eta^{T}(k) \mathcal{A}^{T} P H_{1} G \xi(k) \\
& -2 \beta_{1} \eta^{T}(k) \mathcal{A}^{T} \mathrm{PH}_{1} G C \mathrm{H}_{2} \eta(k) \\
& -2 \beta_{2} \eta^{T}(k) \mathcal{A}^{T} P H_{1} G \bar{s}(k) \\
& -2 \beta_{3} \eta^{T}(k) \mathcal{A}^{T} P H_{1} G U \eta(k) \\
& +2 \mathcal{G}^{T}(k) \bar{W}_{0}^{T} P \bar{W}_{1} \overline{\mathcal{G}}(k) \\
& +2 \mathcal{G}^{T}(k) \bar{W}_{0}^{T} P H_{1} G \xi(k) \\
& -2 \beta_{1} \mathcal{G}^{T}(k) \bar{W}_{0}^{T} P H_{1} G C H_{2} \eta(k) \\
& -2 \beta_{2} \mathcal{G}^{T}(k) \bar{W}_{0}^{T} P H_{1} G \bar{s}(k) \\
& -2 \beta_{3} \mathcal{G}^{T}(k) \bar{W}_{0}^{T} P H_{1} G U \eta(k) \\
& +2 \overline{\mathcal{G}}^{T}(k) \bar{W}_{1}^{T} P H_{1} G \xi(k) \\
& -2 \beta_{1} \overline{\mathcal{G}}^{T}(k) \bar{W}_{1}^{T} P H_{1} G C H_{2} \eta(k) \\
& -2 \beta_{2} \overline{\mathcal{G}}^{T}(k) \bar{W}_{1}^{T} \mathrm{PH} H_{1} G \bar{s}(k) \\
& -2 \beta_{3} \overline{\mathcal{G}}^{T}(k) \bar{W}_{1}^{T} P H_{1} G U \eta(k) \\
& -2 \beta_{1} \xi^{T}(k) G^{T} H_{1}^{T} P H_{1} G C H_{2} \eta(k) \\
& -2 \beta_{2} \xi^{T}(k) G^{T} H_{1}^{T} P H_{1} G \bar{s}(k) \\
& -2 \beta_{3} \xi^{T}(k) G^{T} H_{1}^{T} P H_{1} G U \eta(k) \\
& +2 \beta_{1} \beta_{2} \eta^{T}(k) H_{2}^{T} C^{T} G^{T} H_{1}^{T} P H_{1} G \bar{s}(k) \\
& +2 \beta_{1} \beta_{3} \eta^{T}(k) H_{2}^{T} C^{T} G^{T} H_{1}^{T} P H_{1} G U \eta(k) \\
& +2 \beta_{2} \beta_{3} \bar{s}^{T}(k) G^{T} H_{1}^{T} P H_{1} G U \eta(k) \\
& +2 \tilde{\delta}_{2}^{\alpha}(k) \tilde{\delta}_{3}^{\alpha}(k) \bar{s}^{T}(k) G^{T} H_{1}^{T} P H_{1} G U \eta(k) \\
& +2 \tilde{\delta}_{1}^{\alpha}(k) \tilde{\delta}_{3}^{\alpha}(k) \eta^{T}(k) H_{2}^{T} C^{T} G^{T} H_{1}^{T} P H_{1} G U \eta(k) \\
& +2 \tilde{\delta}_{1}^{\alpha}(k) \tilde{\delta}_{2}^{\alpha}(k) \eta^{T}(k) H_{2}^{T} C^{T} G^{T} H_{1}^{T} P H_{1} G \bar{s}(k) \\
& +\sum_{i=1}^{n} \sum_{j=1}^{n} \eta^{T}(k) Q_{i j} \eta(k)-\eta_{d}^{T}(k) Q \eta_{d}(k) \\
& \left.-\eta^{T}(k) P \eta(k)\right\} \text {. }
\end{aligned}
$$

By noting (3) and (14), the term containing $\tilde{\mathcal{A}}^{T}(k) P \tilde{\mathcal{A}}(k)$ 
can be computed as follows:

$$
\begin{aligned}
& \mathbb{E}\left\{\eta^{T}(k) H_{2}^{T} \tilde{\mathcal{A}}^{T}(k) P \tilde{\mathcal{A}}(k) H_{2} \eta(k)\right\} \\
= & \mathbb{E}\left\{\eta^{T}(k) H_{2}^{T}\left[\begin{array}{l}
\tilde{A}(k) \\
\tilde{A}(k)
\end{array}\right]^{T}\left[\begin{array}{ll}
P_{11} & P_{12} \\
P_{21} & P_{22}
\end{array}\right]\left[\begin{array}{c}
\tilde{A}(k) \\
\tilde{A}(k)
\end{array}\right] H_{2} \eta(k)\right\} \\
= & \mathbb{E}\left\{\eta ^ { T } ( k ) H _ { 2 } ^ { T } \left(\tilde{A}^{T}(k) P_{11} \tilde{A}(k)+2 \tilde{A}^{T}(k) P_{12} \tilde{A}(k)\right.\right. \\
& \left.\left.+\tilde{A}^{T}(k) P_{22} \tilde{A}(k)\right) H_{2} \eta(k)\right\} \\
= & \mathbb{E}\left\{\eta^{T}(k) H_{2}^{T}\left(E \circ\left(P_{11}+P_{12}+P_{21}+P_{22}\right)\right) H_{2} \eta(k)\right\} \\
= & \mathbb{E}\left\{\eta^{T}(k) H_{2}^{T} \tilde{P} H_{2} \eta(k)\right\} .
\end{aligned}
$$

For the term $\bar{w}^{T}(k) \mathcal{B}^{T} P \mathcal{B} \bar{w}(k)$, we have

$$
\begin{aligned}
& \mathbb{E}\left\{\bar{w}^{T}(k) \mathcal{B}^{T} P \mathcal{B} \bar{w}(k)\right\} \\
\leq & \lambda_{\max }\left(\mathcal{B}^{T} P \mathcal{B}\right) \mathbb{E}\left\{w^{T}(k) w(k)+v^{T}(k) v(k)\right\} \\
= & \vartheta
\end{aligned}
$$

By using the elementary inequality $-2 a b \leq a^{2}+b^{2}$, it can be obtained that

$$
\begin{aligned}
& \mathbb{E}\left\{-2 \beta_{3} \eta^{T}(k) \mathcal{A}^{T} P H_{1} G U \eta(k)\right\} \\
& \leq \mathbb{E}\left\{\beta_{3} \eta^{T}(k) \mathcal{A}^{T} P \mathcal{A} \eta(k)\right.\left.+\beta_{3} \eta^{T}(k) U^{T} G^{T} H_{1}^{T} P H_{1} G U \eta(k)\right\}, \\
& \mathbb{E}\left\{-2 \beta_{2} \eta^{T}(k) \mathcal{A}^{T} P H_{1} G \bar{s}(k)\right\} \\
& \leq \mathbb{E}\left\{\beta_{2} \eta^{T}(k) \mathcal{A}^{T} P \mathcal{A} \eta(k)\right. \\
&\left.+\beta_{2} \bar{s}^{T}(k) G^{T} H_{1}^{T} P H_{1} G \bar{s}(k)\right\}, \\
& \mathbb{E}\left\{-2 \beta_{2} \xi^{T}(k) G^{T} H_{1}^{T} P H_{1} G \bar{s}(k)\right\} \\
& \leq \mathbb{E}\left\{\beta_{2} \xi^{T}(k) G^{T} H_{1}^{T} P H_{1} G \xi(k)\right. \\
&\left.+\beta_{2} \bar{s}^{T}(k) G^{T} H_{1}^{T} P H_{1} G \bar{s}(k)\right\}, \\
& \leq \mathbb{E}\left\{-2 \beta_{3} \mathcal{G}^{T}(k) \bar{W}_{0}^{T} P H_{1} G U \eta(k)\right\} \\
& \leq \mathbb{E}\left\{\beta_{3} \mathcal{G}^{T}(k) \bar{W}_{0}^{T} P \bar{W}_{0} \mathcal{G}(k)\right. \\
&\left.+\beta_{3} \eta^{T}(k) U^{T} G^{T} H_{1}^{T} P H_{1} G U \eta(k)\right\}, \\
& \leq \mathbb{E}\left\{-2 \beta_{3} \overline{\mathcal{G}}^{T}(k) \bar{W}_{1}^{T} P H_{1} G U \eta(k)\right\} \\
& \leq \mathbb{E}\left\{\beta_{3} \overline{\mathcal{G}}^{T}(k) \bar{W}_{1}^{T} P \bar{W}_{1} \overline{\mathcal{G}}(k)\right. \\
&\left.+\beta_{3} \eta^{T}(k) U^{T} G^{T} H_{1}^{T} P H_{1} G U \eta(k)\right\}, \\
& \mathbb{E}\left\{-2 \beta_{3} \xi^{T}(k) G^{T} H_{1}^{T} P H_{1} G U \eta(k)\right\} \\
& \leq \mathbb{E}\left\{\beta_{3} \xi^{T}(k) G^{T} H_{1}^{T} P H_{1} G \xi(k)\right. \\
&\left.\quad+\beta_{3} \eta^{T}(k) U^{T} G^{T} H_{1}^{T} P H_{1} G U \eta(k)\right\} .
\end{aligned}
$$

Substituting (30)-(32) into (29) and using Lemma 3 yield

$$
\begin{aligned}
& \mathbb{E}\{V(k+1)-V(k)\} \\
&=\mathbb{E}\left\{\left(1+\beta_{2}+\beta_{3}\right) \eta^{T}(k) \mathcal{A}^{T} P \mathcal{A} \eta(k)\right. \\
&+\left(1+\beta_{3}\right) \mathcal{G}^{T}(k) \bar{W}_{0}^{T} P \bar{W}_{0} \mathcal{G}(k) \\
&+\left(1+\beta_{3}\right) \overline{\mathcal{G}}^{T}(k) \bar{W}_{1}^{T} P \bar{W}_{1} \overline{\mathcal{G}}(k) \\
&+\left(1+\beta_{2}+\beta_{3}\right) \xi^{T}(k) G^{T} H_{1}^{T} P H_{1} G \xi(k) \\
&+\eta^{T}(k) H_{2}^{T} \tilde{P} H_{2} \eta(k) \\
&+\beta_{1} \eta^{T}(k) H_{2}^{T} C^{T} G^{T} H_{1}^{T} P H_{1} G C H_{2} \eta(k) \\
&+3 \beta_{2} \bar{s}^{T}(k) G^{T} H_{1}^{T} P H_{1} G \bar{s}(k) \\
&+5 \beta_{3} \eta^{T}(k) U^{T} G^{T} H_{1}^{T} P H_{1} G U \eta(k) \\
&+2 \eta^{T}(k) \mathcal{A}^{T} P \bar{W}_{0} \mathcal{G}(k)+2 \eta^{T}(k) \mathcal{A}^{T} P \bar{W}_{1} \overline{\mathcal{G}}(k)
\end{aligned}
$$

$$
\begin{aligned}
+ & 2 \eta^{T}(k) \mathcal{A}^{T} P H_{1} G \xi(k) \\
& -2 \beta_{1} \eta^{T}(k) \mathcal{A}^{T} P H_{1} G C H_{2} \eta(k) \\
& +2 \mathcal{G}^{T}(k) \bar{W}_{0}^{T} P \bar{W}_{1} \overline{\mathcal{G}}(k) \\
& +2 \mathcal{G}^{T}(k) \bar{W}_{0}^{T} P H_{1} G \xi(k) \\
& -2 \beta_{1} \mathcal{G}^{T}(k) \bar{W}_{0}^{T} P H_{1} G C H_{2} \eta(k) \\
- & 2 \beta_{2} \mathcal{G}^{T}(k) \bar{W}_{0}^{T} P H_{1} G \bar{s}(k) \\
+ & 2 \overline{\mathcal{G}}^{T}(k) \bar{W}_{1}^{T} P H_{1} G \xi(k) \\
- & 2 \beta_{1} \overline{\mathcal{G}}^{T}(k) \bar{W}_{1}^{T} P H_{1} G C H_{2} \eta(k) \\
- & 2 \beta_{2} \overline{\mathcal{G}}^{T}(k) \bar{W}_{1}^{T} P H_{1} G \bar{s}(k) \\
- & 2 \beta_{1} \xi^{T}(k) G^{T} H_{1}^{T} P H_{1} G C H_{2} \eta(k) \\
& +\sum_{i=1}^{n} \sum_{j=1}^{n} \eta^{T}(k) Q_{i j} \eta(k) \\
& \left.-\eta_{d}^{T}(k) Q \eta_{d}(k)-\eta^{T}(k) P \eta(k)+\vartheta\right\} \\
\leq & \mathbb{E}\left\{\zeta^{T}(k) \bar{\Phi} \zeta(k)+\vartheta\right\}
\end{aligned}
$$

where

$$
\begin{aligned}
& \zeta(k)=\left[\begin{array}{llllll}
\eta^{T}(k) & \mathcal{G}^{T}(k) & \overline{\mathcal{G}}^{T}(k) & \xi^{T}(k) & \bar{s}^{T}(k) & \eta_{d}^{T}(k)
\end{array}\right]^{T} \\
& \bar{\Phi}=\left[\begin{array}{cccccc}
\bar{\Theta}_{11} & \Theta_{12} & \Theta_{13} & \Theta_{14} & 0 & 0 \\
* & \bar{\Theta}_{22} & \Theta_{23} & \Theta_{24} & \Theta_{25} & 0 \\
* & * & \bar{\Theta}_{33} & \Theta_{34} & \Theta_{35} & 0 \\
* & * & * & \bar{\Theta}_{44} & 0 & 0 \\
* & * & * & * & \bar{\Theta}_{55} & 0 \\
* & * & * & * & * & -Q
\end{array}\right], \\
& \bar{\Theta}_{11}=\left(1+\beta_{2}+\beta_{3}\right) \mathcal{A}^{T} P \mathcal{A}+H_{2}^{T} \tilde{P} H_{2} \\
& +5 \beta_{3} U^{T} G^{T} H_{1}^{T} P H_{1} G U \\
& +\beta_{1} H_{2}^{T} C^{T} G^{T} H_{1}^{T} P H_{1} G C H_{2}-\beta_{1} \mathcal{A}^{T} P H_{1} G C H_{2} \\
& -\beta_{1} H_{2}^{T} C^{T} G^{T} H_{1}^{T} P \mathcal{A}-P+\sum_{i=1}^{n} \sum_{j=1}^{n} Q_{i j}, \\
& \bar{\Theta}_{22}=\left(1+\beta_{3}\right) \bar{W}_{0}^{T} P \bar{W}_{0}, \\
& \bar{\Theta}_{44}=\left(1+\beta_{2}+\beta_{3}\right) G^{T} H_{1}^{T} P H_{1} G \text {, } \\
& \bar{\Theta}_{33}=\left(1+\beta_{3}\right) \bar{W}_{1}^{T} P \bar{W}_{1}, \quad \bar{\Theta}_{55}=3 \beta_{2} G^{T} H_{1}^{T} P H_{1} G .
\end{aligned}
$$

Under the triggering condition, it follows from (18), (19) and (20) that

$$
\begin{aligned}
\mathbb{E} & \{V(k+1)-V(k)\} \\
\leq & \mathbb{E}\left\{\zeta^{T}(k) \bar{\Phi} \zeta(k)-\lambda_{1}\left(\mathcal{G}^{T}(k) \mathcal{G}(k)-\eta^{T}(k) \bar{M} \eta(k)\right)\right. \\
& -\lambda_{2}\left(\overline{\mathcal{G}}^{T}(k) \overline{\mathcal{G}}(k)-\eta_{d}^{T}(k) \hat{M} \eta_{d}(k)\right) \\
& -\lambda_{3}\left(\bar{s}^{T}(k) \bar{s}(k)-\eta^{T}(k) H_{2}^{T} C^{T}\left(K^{T}+I\right) \bar{s}(k)\right. \\
& \left.+\eta^{T}(k) H_{2}^{T} C^{T} K^{T} C H_{2} \eta(k)\right) \\
& \left.-\lambda_{4}\left(\xi^{T}(k) \xi(k)-\delta\right)+\vartheta\right\} \\
= & \left.\mathbb{E}\left\{\zeta^{T}(k) \Phi \zeta(k)\right\}\right\}+\lambda_{4} \delta+\vartheta \\
\leq & -\lambda_{\min }(-\Phi) \mathbb{E}\left\{\eta^{T}(k) \eta(k)\right\}+\lambda_{4} \delta+\vartheta .
\end{aligned}
$$

Next, let's estimate the upper bound of $\mathbb{E}\left\{\|\eta(T)\|^{2}\right\}$. According to the definition of functional $V(k)$, it can be obtained that

$$
\begin{aligned}
V(k) \leq & \lambda_{\max }(P)\|\eta(k)\|^{2} \\
& +\sum_{i=1}^{n} \sum_{j=1}^{n} \lambda_{\max }\left(Q_{i j}\right) \sum_{l=k-\tau_{i j}}^{k-1}\|\eta(l)\|^{2} .
\end{aligned}
$$


Introducing a scalar $\alpha>1$, we compute

$$
\begin{aligned}
& \mathbb{E}\left\{\alpha^{k+1} V(k+1)-\alpha^{k} V(k)\right\} \\
= & \mathbb{E}\left\{\alpha^{k+1}(V(k+1)-V(k))+\alpha^{k}(\alpha-1) V(k)\right\} \\
\leq & \alpha^{k} \phi(\alpha) \mathbb{E}\left\{\|\eta(k)\|^{2}\right\}+\alpha^{k+1}\left(\lambda_{4} \delta+\vartheta\right) \\
& +\alpha^{k} \sum_{i=1}^{n} \sum_{j=1}^{n} \varphi_{i j}(\alpha) \sum_{l=k-\tau_{i j}}^{k-1} \mathbb{E}\left\{\|\eta(l)\|^{2}\right\} .
\end{aligned}
$$

where $\phi(\alpha)$ and $\varphi_{i j}(\alpha)$ are defined in (25).

Denote $d=\max _{1 \leq i, j \leq n}\left\{\tau_{i j}\right\}$. For any integer $T \geq d+1$, summing up both sides of (40) from 0 to $T-1$ with respect to $k$, we have

$$
\begin{aligned}
& \mathbb{E}\left\{\alpha^{T} V(T)\right\}-\mathbb{E}\{V(0)\} \\
\leq & \phi(\alpha) \sum_{k=0}^{T-1} \alpha^{k} \mathbb{E}\left\{\|\eta(k)\|^{2}\right\}+\frac{\alpha\left(1-\alpha^{T}\right)}{1-\alpha}\left(\lambda_{4} \delta+\vartheta\right) \\
& +\sum_{i=1}^{n} \sum_{j=1}^{n} \varphi_{i j}(\alpha) \sum_{k=0}^{T-1} \sum_{l=k-\tau_{i j}}^{k-1} \alpha^{k} \mathbb{E}\left\{\|\eta(l)\|^{2}\right\} .
\end{aligned}
$$

For the last term in (41), we further have

$$
\begin{aligned}
& \sum_{k=0}^{T-1} \sum_{l=k-\tau_{i j}}^{k-1} \alpha^{k} \mathbb{E}\left\{\|\eta(l)\|^{2}\right\} \\
\leq & \left(\sum_{l=-\tau_{i j}}^{-1} \sum_{k=0}^{l+\tau_{i j}}+\sum_{l=0}^{T-\tau_{i j}-1} \sum_{k=l+1}^{l+\tau_{i j}}\right. \\
& \left.+\sum_{l=T-\tau_{i j}}^{T-1} \sum_{k=l+1}^{T-1}\right) \alpha^{k} \mathbb{E}\left\{\|\eta(l)\|^{2}\right\} \\
\leq & \frac{\alpha^{\tau_{i j}}-1}{\alpha-1} \sum_{l=-\tau_{i j}}^{-1} \mathbb{E}\left\{\|\eta(l)\|^{2}\right\} \\
& +\frac{\alpha\left(\alpha^{\tau_{i j}}-1\right)}{\alpha-1} \sum_{l=0}^{T-1} \alpha^{l} \mathbb{E}\left\{\|\eta(l)\|^{2}\right\} \\
& +\frac{\alpha\left(\alpha^{\tau_{i j}-1}-1\right)}{\alpha-1} \sum_{l=0}^{T-1} \alpha^{l} \mathbb{E}\left\{\|\eta(l)\|^{2}\right\} .
\end{aligned}
$$

From (41) and (42), it is easily known that

$$
\begin{aligned}
& \mathbb{E}\left\{\alpha^{T} V(T)\right\}-\mathbb{E}\{V(0)\} \\
\leq & \phi(\alpha) \sum_{k=0}^{T-1} \alpha^{k} \mathbb{E}\left\{\|\eta(k)\|^{2}\right\}+\frac{\alpha\left(1-\alpha^{T}\right)}{1-\alpha}\left(\lambda_{4} \delta+\vartheta\right) \\
& +\sum_{i=1}^{n} \sum_{j=1}^{n} \varphi_{i j}(\alpha)\left(\frac{\alpha^{\tau_{i j}}-1}{\alpha-1} \sum_{l=-\tau_{i j}}^{-1} \mathbb{E}\left\{\|\eta(l)\|^{2}\right\}\right. \\
& +\frac{\alpha\left(\alpha^{\tau_{i j}}-1\right)}{\alpha-1} \sum_{l=0}^{T-1} \alpha^{l} \mathbb{E}\left\{\|\eta(l)\|^{2}\right\} \\
& \left.+\frac{\alpha\left(\alpha^{\tau_{i j}}-1-1\right)}{\alpha-1} \sum_{l=0}^{T-1} \alpha^{l} \mathbb{E}\left\{\|\eta(l)\|^{2}\right\}\right)
\end{aligned}
$$

$$
\begin{aligned}
\leq & \zeta(\alpha) \sum_{k=0}^{T-1} \alpha^{k} \mathbb{E}\left\{\|\eta(k)\|^{2}\right\}+\frac{\alpha\left(1-\alpha^{T}\right)}{1-\alpha}\left(\lambda_{4} \delta+\vartheta\right) \\
& +\frac{1}{\alpha-1} \sum_{i=1}^{n} \sum_{j=1}^{n} \varphi_{i j}(\alpha) \tau_{i j}\left(\alpha^{\tau_{i j}}-1\right) \\
& \times \max _{-\tau_{i j} \leq l \leq 0} \mathbb{E}\left\{\|\eta(l)\|^{2}\right\}
\end{aligned}
$$

where

$$
\begin{aligned}
\zeta(\alpha)= & \phi(\alpha)+\frac{1}{\alpha-1} \sum_{i=1}^{n} \sum_{j=1}^{n} \varphi_{i j}(\alpha) \\
& \times\left(\alpha^{\tau_{i j}+1}+\alpha^{\tau_{i j}}-2 \alpha\right) .
\end{aligned}
$$

Note that $\zeta(1)=-\lambda_{\min }(-\Phi)<0$ and $\lim _{\alpha \rightarrow+\infty} \zeta(\alpha)=$ $+\infty$. Therefore, there exists a scalar $\alpha_{0}>1$ such that $\zeta\left(\alpha_{0}\right)=$ 0 . Then, it follows from (43) that

$$
\begin{aligned}
& \mathbb{E}\left\{\alpha_{0}^{T} V(T)\right\}-\mathbb{E}\{V(0)\} \\
\leq & \frac{\alpha_{0}\left(1-\alpha_{0}^{T}\right)}{1-\alpha_{0}}\left(\lambda_{4} \delta+\vartheta\right) \\
& +\frac{1}{\alpha_{0}-1} \sum_{i=1}^{n} \sum_{j=1}^{n} \tau_{i j} \varphi_{i j}\left(\alpha_{0}\right) \\
& \times\left(\alpha_{0}^{\tau_{i j}}-1\right) \max _{-\tau_{i j} \leq l \leq 0} \mathbb{E}\left\{\|\eta(l)\|^{2}\right\}
\end{aligned}
$$

\section{Considering}

$$
\begin{aligned}
& \mathbb{E}\{V(0)\} \\
\leq & \lambda_{\max }(P) \mathbb{E}\left\{\|\eta(0)\|^{2}\right\} \\
& +\sum_{i=1}^{n} \sum_{j=1}^{n} \lambda_{\max }\left(Q_{i j}\right) \sum_{l=-\tau_{i j}}^{-1}\|\eta(l)\|^{2} \\
\leq & \sum_{i=1}^{n} \sum_{j=1}^{n} \frac{1}{n^{2}} \lambda_{\max }(P) \max _{-\tau_{i j} \leq l \leq 0} \mathbb{E}\left\{\|\eta(l)\|^{2}\right\} \\
& +\sum_{i=1}^{n} \sum_{j=1}^{n} \tau_{i j} \lambda_{\max }\left(Q_{i j}\right) \max _{-\tau_{i j} \leq l \leq 0} \mathbb{E}\left\{\|\eta(l)\|^{2}\right\} \\
\leq & \sum_{i=1}^{n} \sum_{j=1}^{n} \max \left(\frac{1}{n^{2}} \lambda_{\max }(P), \tau_{i j} \lambda_{\max }\left(Q_{i j}\right)\right) \\
& \times \max _{-\tau_{i j} \leq l \leq 0} \mathbb{E}\left\{\|\eta(l)\|^{2}\right\}
\end{aligned}
$$

and

$$
\mathbb{E}\left\{\alpha_{0}^{T} V(T)\right\} \geq \lambda_{\min }(P) \alpha_{0}^{T} \mathbb{E}\left\{\|\eta(T)\|^{2}\right\},
$$

we have

$$
\begin{aligned}
\mathbb{E}\left\{\|\eta(T)\|^{2}\right\} \leq & \frac{\alpha_{0}^{T}-1}{\alpha_{0}^{T-1}\left(\alpha_{0}-1\right) \lambda_{\min }(P)}\left(\lambda_{4} \delta+\vartheta\right) \\
& +\frac{\varpi\left(\alpha_{0}\right)}{\alpha_{0}^{T} \lambda_{\min }(P)}
\end{aligned}
$$

where

$$
\begin{aligned}
\varpi\left(\alpha_{0}\right)= & \sum_{i=1}^{n} \sum_{j=1}^{n}\left(\frac{\tau_{i j} \varphi_{i j}\left(\alpha_{0}\right)\left(\alpha_{0}^{\tau_{i j}}-1\right)}{\alpha_{0}-1}\right. \\
& \left.+\max \left(\frac{1}{n^{2}} \lambda_{\max }(P), \tau_{i j} \lambda_{\max }\left(Q_{i j}\right)\right)\right) \\
& \times \max _{-\tau_{i j} \leq l \leq 0} \mathbb{E}\left\{\|\eta(l)\|^{2}\right\} .
\end{aligned}
$$


By taking $\mu=\frac{1}{\alpha_{0}}, \kappa(T)=\frac{\alpha_{0}^{T}-1}{\alpha_{0}^{T-1}\left(\alpha_{0}-1\right) \lambda_{\min }(P)}\left(\lambda_{4} \delta+\vartheta\right)$, and $\nu=\frac{\varpi\left(\alpha_{0}\right)}{\lambda_{\min }(P)}$, it follows easily from Definition 1 that the augmented system (13) is exponentially mean-square ultimately bounded and the ultimate bounded is given by

$$
\bar{\kappa}=\lim _{T \rightarrow+\infty} \kappa(T)=\frac{\alpha_{0}}{\left(\alpha_{0}-1\right) \lambda_{\min }(P)}\left(\lambda_{4} \delta+\vartheta\right) .
$$

The proof of Theorem 1 is complete.

\section{APPENDIX II}

\section{PROOF OF THEOREM 2}

Proof: In terms of Theorem 1, we just need to show that inequality (26) in Theorem 2 holds implies inequality (22) in Therem 1 holds.

By using the Schur complement lemma, it is easily known that $\Phi<0$ is equivalent to

$$
\begin{aligned}
& \tilde{\Psi}= \\
& {\left[\begin{array}{ccccccccc}
\bar{\Xi}_{11} & 0 & \Xi_{13} & 0 & \bar{\Xi}_{15} & \bar{\Xi}_{16} & \bar{\Xi}_{17} & 0 & 0 \\
* & \Xi_{22} & \bar{\Xi}_{23} & 0 & \bar{\Xi}_{25} & 0 & 0 & \bar{\Xi}_{28} & 0 \\
* & * & \Xi_{33} & 0 & 0 & 0 & 0 & 0 & \bar{\Xi}_{39} \\
* & * & * & \Xi_{44} & 0 & 0 & 0 & 0 & 0 \\
* & * & * & * & \Xi_{55} & 0 & 0 & 0 & 0 \\
* & * & * & * & * & \Xi_{66} & 0 & 0 & 0 \\
* & * & * & * & * & * & \Xi_{77} & 0 & 0 \\
* & * & * & * & * & * & * & \Xi_{88} & 0 \\
* & * & * & * & * & * & * & * & \Xi_{99}
\end{array}\right]} \\
& <0
\end{aligned}
$$

where

$$
\begin{aligned}
\Xi_{11}= & H_{2}^{T} \tilde{P} H_{2}-P+\lambda_{1} \bar{M}+\sum_{i=1}^{n} \sum_{j=1}^{n} Q_{i j} \\
& -\lambda_{3} H_{2}^{T} C^{T} K C H_{2}, \\
\bar{\Xi}_{15}= & \mathcal{A}^{T} P-\beta_{1} H_{2}^{T} C^{T} G^{T} H_{1}^{T} P, \quad \bar{\Xi}_{17}=\sqrt{5 \beta_{3}} U^{T} G^{T} H_{1}^{T} P, \\
\bar{\Xi}_{16}= & {\left[\begin{array}{lll}
\sqrt{\beta_{2}+\beta_{3}} \mathcal{A}^{T} P & \sqrt{\beta_{1}\left(1-\beta_{1}\right)} H_{2}^{T} C^{T} G^{T} H_{1}^{T} P
\end{array}\right], } \\
\bar{\Xi}_{23}= & {\left[\begin{array}{lll}
-\beta_{2} G^{T} H_{1}^{T} P \bar{W}_{0} & -\beta_{2} G^{T} H_{1}^{T} P \bar{W}_{1} & 0
\end{array}\right]^{T}, } \\
\bar{\Xi}_{25}= & {\left[\begin{array}{lll}
P \bar{W}_{0} & P \bar{W}_{1} & P H_{1} G
\end{array}\right]^{T}, \quad \bar{\Xi}_{39}=\sqrt{3 \beta_{2}} G^{T} H_{1}^{T} P, } \\
\bar{\Xi}_{28}= & {\left[\begin{array}{lll}
0 & 0 & \sqrt{\beta_{2}+\beta_{3}} P H_{1} G
\end{array}\right]^{T} . }
\end{aligned}
$$

Let's now deal with the uncertainty induced by quantization effect. Set

$$
\begin{aligned}
T & =\left[\begin{array}{llllllllll}
0 & 0 & 0 & 0 & 0 & 0 & \sqrt{5 \beta_{3}} G^{T} H_{1}^{T} P & 0 & 0
\end{array}\right]^{T}, \\
N & =\left[\begin{array}{llllllllll}
\Lambda_{q} C H_{2} & 0 & 0 & 0 & 0 & 0 & 0 & 0 & 0
\end{array}\right] .
\end{aligned}
$$

The matrix $\tilde{\Psi}$ can then be written as

$$
\tilde{\Psi}=\bar{\Psi}+T F N+(T F N)^{T}
$$

where

$\bar{\Psi}=\left[\begin{array}{ccccccccc}\bar{\Xi}_{11} & 0 & \Xi_{13} & 0 & \bar{\Xi}_{15} & \bar{\Xi}_{16} & \tilde{\Xi}_{17} & 0 & 0 \\ * & \Xi_{22} & \bar{\Xi}_{23} & 0 & \bar{\Xi}_{25} & 0 & 0 & \bar{\Xi}_{28} & 0 \\ * & * & \Xi_{33} & 0 & 0 & 0 & 0 & 0 & \bar{\Xi}_{39} \\ * & * & * & \Xi_{44} & 0 & 0 & 0 & 0 & 0 \\ * & * & * & * & \Xi_{55} & 0 & 0 & 0 & 0 \\ * & * & * & * & * & \Xi_{66} & 0 & 0 & 0 \\ * & * & * & * & * & * & \Xi_{77} & 0 & 0 \\ * & * & * & * & * & * & * & \Xi_{88} & 0 \\ * & * & * & * & * & * & * & * & \Xi_{99}\end{array}\right]$

with $\tilde{\Xi}_{17}=\sqrt{5 \beta_{3}} H_{2}^{T} C^{T} G^{T} H_{1}^{T} P$.

By using Lemma 4 and the Schur complement lemma again, it is shown that $\tilde{\Psi}<0$ if and only if

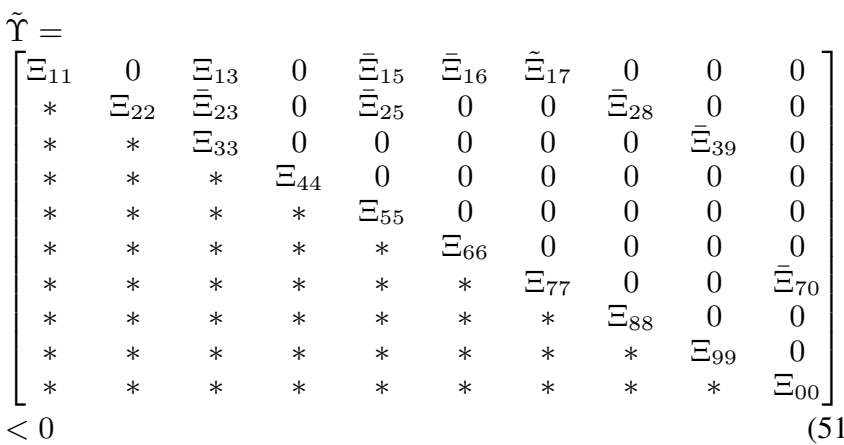

where $\bar{\Xi}_{70}=\sqrt{5 \beta_{3}} P H_{1} G$.

By considering the relation $Y=P_{22} G$, it can be seen that matrix $\Upsilon$ defined in Theorem 2 is the exactly same as matrix $\tilde{\Upsilon}$ given in (51) which means that inequality (26) holds implies inequality (22) holds. Therefore, the rest of the proof follows from that of Theorem 1 immediately.

\section{REFERENCES}

[1] H. Azad, S. Mekhilef, and V. Ganapathy, "Long-term wind speed forecasting and general pattern recognition using neural networks," IEEE Trans. Sustainable Energy, vol. 5, pp. 546-553, Apr. 2014.

[2] H. Chaoui, P. Sicard, and W. Gueaieb, "ANN-based adaptive control of robotic manipulators with friction and joint elasticity," IEEE Trans. Industrial Electronics, Vol. 56, pp. 3174-3187, Aug. 2009.

[3] Y. Chen and W. Zheng, "Stochastic state estimation for neural networks with distributed delays and Markovian jump," Neural Networks, vol. 25, no. 1, pp. 14-20, 2012.

[4] D. V. Dimarogonas, E. Frazzoli, and K. H. Johansson, "Distributed event-triggered control for multi-agent systems," IEEE Trans. Automatic Control, vol. 57, pp. 1291-1297, May. 2012.

[5] D. Ding, Z. Wang, B. Shen, and H. Dong, "Envelope-constrained $H_{\infty}$ filtering with fading measurements and randomly occurring nonlinearities: the finite horizon case," Automatica, vol. 55, no. 5, pp. 37-45, 2015.

[6] D. Ding, Z. Wang, B. Shen, and G. Wei, "Event-triggered consensus control for discrete-time stochastic multi-agent systems: the input-tostate stability in probability," Automatica, vol. 62, no. 12, pp. 284-291. 2015.

[7] H. Dong, Z. Wang, F. E. Alsaadi, and B. Ahmad, "Event-triggered robust distributed state estimation for sensor networks with state-dependent noises," International Journal of General Systems, vol. 44, no. 2, pp. 254-266, 2015.

[8] T. El-Midany, M. El-Baz, and M. Abd-Elwahed, "A proposed framework for control chart pattern recognition in multivariate process using artificial neural networks," Expert Systems with Applications, vol. 37, no. 2, pp. 1035-1042, 2010.

[9] Y. Fan, G. Feng, Y. Wang, and C. Song, "Distributed event-triggered control of multi-agent systems with combinational measurements," $\mathrm{Au}$ tomatica, vol. 49 , no. 2, pp. 671-675, 2012 
[10] Z. Feng and J. Lam, "Stability and dissipativity analysis of distributed delay cellular neural networks," IEEE Trans. Neural Networks, vol. 22, pp. 976-981, Jun. 2011.

[11] M. Fu and L. Xie, "The sector bound approach to quantized feedback control," IEEE Trans. Automatic Control, vol. 50, pp. 1689-1711, Nov. 2005.

[12] A. Graves, M. Liwicki, S. Fernandez, R. Bertolami, H. Bunke, and J. Schmidhuber, "A novel connectionist system for improved unconstrained handwriting recognition," IEEE Trans. Pattern Analysis and Machine Intelligence, vol. 31, pp. 855-868, May 2009.

[13] X. He, Z. Wang, and D. Zhou, "Robust $H_{\infty}$ filtering for networked systems with multiple state delays," International Journal of Control, vol. 80, no. 8, pp. 1217-1232, 2007.

[14] J. J. Hopfield and D. W. Tank, "“Neural” computation of decisions optimization problems," Biological Cybern., vol. 52, no. 3, pp. 141-152, 1985.

[15] F. O. Hounkpevi and E. E. Yaz, "Robust minimum variance linear state estimators for multiple sensors with different failure rates," Automatica, vol. 43, no. 7, pp. 1274-1280, 2007.

[16] S. Hsu and W. Zhu, "Intelligent data analysis using artificial neural networks," Research Quarterly for Exercise and Sport, vol. 73, no. 1, pp. A38-A39, 2002.

[17] H. Hu and P. Woo, "Fuzzy supervisory sliding-mode and neural-network control for robotic manipulators," IEEE Trans. Industrial Electronics, vol. 53, pp. 929-940, Jun. 2006.

[18] H. Huang, T. Huang, and X. Chen, "Guaranteed $H_{\infty}$ performance state estimation of delayed static neural networks," IEEE Trans. Circuits and Systems II: Express Briefs, vol. 60, pp. 371-375, Jun. 2013.

[19] M. Khashei, A. Hamadani, and M. Bijari, "A fuzzy intelligent approach to the classification problem in gene expression data analysis," Knowledge-Based Systems, vol. 27, no. 3, pp. 465-474, 2012.

[20] S. Kluge, K. Reif, and M. Brokate, "Stochastic stability of the extended Kalman filter with intermittent observations," IEEE Trans. Automatic Control, vol. 55, pp. 514-518, Feb. 2010.

[21] R. Kozma and M. Puljic, "Hierarchical random cellular neural networks for system-level brain-like signal processing," Neural Networks, vol. 45, no. 9, pp. 101-110, 2013.

[22] L. Li, D. W. C. Ho, and J. Lu, "A unified approach to practical consensus with quantized data and time delay," IEEE Trans. Circuits and Systems-I: Regular Paper, vol. 60, pp. 2668-2678, Oct. 2013.

[23] Q. Li, B. Shen, J. Liang, and H. Shu, "Event-triggered synchronization control for complex networks with uncertain inner coupling," International Journal of General Systems, vol. 44, no. 2, pp. 212-225, 2015.

[24] B. Liu, W. Lu, and T. Chen, "Global almost sure self-synchronization of Hopfield neural networks with randomly switching connections," Neural Networks, vol. 24, no. 3, pp. 305-310, 2011

[25] Q. Liu, Z. Wang, X. He, and D. H. Zhou, "Event-based $H_{\infty}$ consensus control of multi-agent systems with relative output feedback: the finite-horizon case," IEEE Transactions on Automatic Control, vol. 60, pp. 2553-2558, Sep. 2015.

[26] Y. Liu, F. E. Alsaadi, X. Yin, and Y. Wang, "Robust $H_{\infty}$ filtering for discrete nonlinear delayed stochastic systems with missing measurements and randomly occurring nonlinearities," International Journal of General Systems, vol. 44, no. 2, pp. 169-181, 2015

[27] Y. Liu, Y. Wang, X. Zhu, and X. Liu, "Optimal guaranteed cost control of a class of hybrid systems with mode-dependent mixed time delays," International Journal of Systems Science, vol. 45, no. 7, pp. 1528-1538, 2014.

[28] J. Lu and J. Cao, "Adaptive synchronization of uncertain dynamical networks with delayed coupling," Nonlinear Dynamics, vol. 53, nos. 12, pp. 107-115, 2008

[29] Y. Luo, G. Wei, Y. Liu, and X. Ding, "Reliable $H_{\infty}$ state estimation for 2-D discrete systems with infinite distributed delays and incomplete observations," International Journal of General Systems, vol. 44, no. 2, pp. 155-168, 2015.

[30] A. N. Michel, J. A. Farrell, and W. Porod, "Qualitative analysis of neural networks," IEEE Trans. Circuits and Systems, vol. 36, pp. 229-243, Feb. 1989

[31] S. Mou, H. Gao, W. Qiang, and Z. Fei, "State estimation for discretetime neural networks with time-varying delays," Neurocomputing, vol. 72, nos. 1-3, pp. 643-647, 2008.

[32] H. Qiao, J. Peng, and Z. Xu, "Nonlinear measures: A new approach to exponential stability analysis for Hopfield-type neural networks," IEEE Trans. Neural Networks, vol. 12, pp. 360-370, Mar. 2001.

[33] B. Shen, Z. Wang, D. Ding, and H. Shu, " $H_{\infty}$ state estimation for complex networks with uncertain inner coupling and incomplete measurements," IEEE Trans. Neural Networks and Learning Systems, vol, 24, pp. 2027-2037, Dec. 2013.

[34] D. W. Tank and J. J. Hopfield, "Simple "Neural" optimization networks: An A/D converter, signal decision circuit, and a linear programming circuit," IEEE Trans. Circuits and Systems, vol. CAS-33, pp. 533-541, May. 1986.

[35] E. Tian, D. Yue, and C. Peng, "Quantized output feedback control for networked control systems," Information Sciences, vol. 178, no. 12, pp. 2734-2749, 2008

[36] Z. Wang, D. W. C. Ho, and X. Liu, "State estimation for delayed neural networks," IEEE Trans. Neural Networks, vol. 16, pp. 279-284, Jan. 2005.

[37] Z. Wang, J. Lam, and X. Liu, "Filtering for a class of nonlinear discretetime stochastic systems with state delays," Journal of Computational and Applied Mathematics, vol, 201, no. 1, pp. 153-163, 2007.

[38] G. Wei, F. Han, L. Wang, and Y. Song, "Reliable $H_{\infty}$ filtering for discrete piecewise linear systems with infinite distributed delays," International Journal of General Systems, vol. 43, nos. 3-4, pp. 346358,2014

[39] K. Watabe, K. Shimizu, and M. Yoneyama, "Millimeter-wave active imaging using neural networks for signal processing," IEEE Trans. Microwave Theory and Techniques, vol. 51, pp. 1512-1516, May 2003.

[40] L. Wu, Z. Feng, and W. Zheng, "Exponential stability analysis for delayed neural networks with switching parameters: Average dwell time approach," IEEE Trans. Neural Networks, vol. 21, pp. 1396-1407, Sep. 2010

[41] Y. Xiao, Y. Cao, and Z. Lin, "Robust filtering for discrete-time systems with saturation and its application to transmultiplexers," IEEE Trans. Signal Processing, vol. 52, pp. 1266-1277, May. 2004.

[42] S. Xu and J. Lam, "Robust output feedback stabilization of uncertain discrete time-delay stochastic systems with multiplicative noise," Dynamics Continuous Discrete Impulsive Systems, vol. 12, no. 1, pp. 41-58, 2005.

[43] F. Yang and Y. Li, "Set-membership filtering for systems with sensor saturation," Automatica, vol. 45, no. 8, pp. 1896-1902, 2009.

[44] D. Zhang and L. Yu, "Exponential state estimation for Markovian jumping neural networks with time-varying discrete and distributed delays," Neural Networks, vol. 35, no. 11, pp. 103-111, 2012.

[45] Z. Zuo, C. Yang, and Y. Wang, "A new method for stability analysis of recurrent neural networks with interval time-varying delay," IEEE Trans. Neural Networks, vol. 21, pp. 339-3445, Feb. 2010.

[46] G. Zhang and Y. Shen, "New algebraic criteria for synchronization stability of chaotic memristive neural networks with time-varying delays,' IEEE Trans. Neural Networks and Learning Systems, vol. 24, pp. 17011707, Oct. 2013.

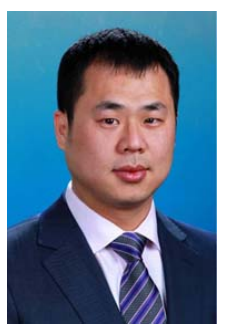

Bo Shen (M'12) received his B.Sc. degree in mathematics from Northwestern Polytechnical University, Xi'an, China, in 2003 and the Ph.D. degree in control theory and control engineering from Donghua University, Shanghai, China, in 2011.

$\mathrm{He}$ is currently a Professor with the School of Information Science and Technology, Donghua University, Shanghai, China. From 2009 to 2010, he was a Research Assistant in the Department of Electrical and Electronic Engineering, the University of Hong Kong, Hong Kong. From 2010 to 2011, he was a Visiting PhD Student in the Department of Information Systems and Computing, Brunel University, U.K. From 2011 to 2013, he was a Research Fellow (Scientific co-worker) in the Institute for Automatic Control and Complex Systems, University of Duisburg-Essen, Germany. Prof. Shen's research interests include nonlinear control and filtering, stochastic control and filtering, as well as complex networks and neural networks. He has published around 40 papers in refereed international journals.

Prof. Shen is serving as an Associate Editor or Editorial Board Member for five international journals, including Systems Science and Control Engineering, Journal of The Franklin Institute, Circuits, Systems, and Signal Processing, Neurocomputing, and Mathematical Problems in Engineering. He is a very active reviewer for many international journals. 


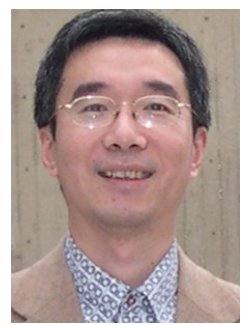

Zidong Wang (SM'03-F'14) was born in Jiangsu, China, in 1966. He received the B.Sc. degree in mathematics from Suzhou University, Suzhou, China, in 1986 and the M.Sc. and Ph.D. degrees in applied mathematics and electrical engineering from the Nanjing University of Science and Technology, Nanjing, China, in 1990 and 1994, respectively.

$\mathrm{He}$ is currently a Professor of Dynamical Systems and Computing with the Department of Computer Science, Brunel University London, Uxbridge, U.K. From 1990 to 2002, he held teaching and research appointments in universities in China, Germany, and the U.K. His current research interests include dynamical systems, signal processing, bioinformatics, control theory, and applications. He has published over 200 papers in refereed international journals.

Prof. Wang was the recipient of the Alexander von Humboldt Research Fellowship of Germany, the JSPS Research Fellowship of Japan, the William Mong Visiting Research Fellowship of Hong Kong. He serves as an Associate Editor for 12 international journals, including IEEE Transactions on Automatic Control, IEEE Transactions on Control Systems Technology, IEEE Transactions on Neural Networks, IEEE Transactions on Signal Processing, and IEEE Transactions on Systems, Man, and Cybernetics-Part C. He is a fellow of the Royal Statistical Society and a Program Committee Member for many international conferences.

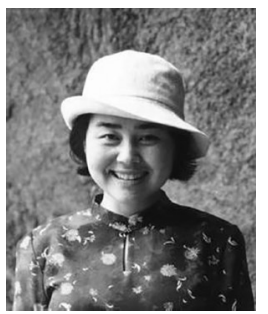

Hong Qiao (SM'06) received the B.Eng. degree in hydraulics and control and the M.Eng. degree in robotics and automation from Xi'an Jiaotong University, and the Ph.D. degree in robotics control from De Montfort University, Leicester, U.K., in 1995.

She was an Assistant Professor with the City University of Hong Kong, Hong Kong, and a Lecturer with the University of Manchester, Manchester, U.K., from 1997 to 2004. She is currently a Professor with the State Key Laboratory of Management and Control for Complex Systems, Institute of Automation, Chinese Academy of Sciences, Beijing, China. Her current research interests include robotics, machine learning, and pattern recognition. 